\title{
Pauli-Lubanski limit and stress-energy tensor for infinite-spin fields
}

\author{
Karl-Henning Rehren \\ Institute for Theoretical Physics, Georg-August-University Göttingen, \\ Friedrich-Hund-Platz 1, 3707 ry Göttingen, Germany \\ E-mail: rehren@theorie.physik.uni-goettingen.de
}

ABSTRACT: String-localized quantum fields transforming in Wigner's infinite-spin representations were originally introduced in $[18,19]$. We construct these fields as limits of fields of finite mass $m \rightarrow 0$ and finite spin $s \rightarrow \infty$. We determine a string-localized infinite-spin quantum stress-energy tensor with a novel prescription that does not refer to a classical Lagrangean.

KeYwords: Higher Spin Symmetry, Space-Time Symmetries

ARXIV EPRINT: 1709.04858

Dedicated to Klaus Fredenhagen on the occasion of his 70th birthday 


\section{Contents}

1 Introduction $\quad 1$

1.1 Quantum fields in the infinite-spin representations 1

1.2 Contents and plan of the paper 5

2 String-localized infinite-spin fields $\quad 6$

2.1 The scalar standard field 8

2.2 Tensor fields I 10

2.3 Tensor fields II 11

3 Preparations for the Pauli-Lubanski limit $\quad 13$

$\begin{array}{ll}3.1 \text { Two-point functions and commutators: basics } & 13\end{array}$

$\begin{array}{lll}3.2 & \text { Two-point functions of string-localized spin } 1 \text { fields } & 14\end{array}$

$\begin{array}{lll}3.3 & \text { Two-point functions of infinite-spin fields } & 15\end{array}$

$\begin{array}{ll}3.4 \text { Massive fields of finite spin: definitions and properties } & 16\end{array}$

4 The Pauli-Lubanski limit $\quad 18$

5 Stress-energy tensors $\quad 21$

5.1 Quantum stress-energy tensors 22

$\begin{array}{lll}5.2 & \text { Proof of Proposition } 5.1 & 25\end{array}$

5.3 Properties of the infinite-spin stress-energy tensor 27

$\begin{array}{lll}\text { 5.3.1 Matrix elements } & 27\end{array}$

5.3.2 Two-point functions and vacuum fluctuations 28

$\begin{array}{lll}\text { 5.3.3 Commutators } & 29\end{array}$

5.3.4 Thermal states: equation of state and equipartition 30

6 Conclusion 31

A Pauli-Lubanski limit of Wigner representations 32

\section{Introduction}

\subsection{Quantum fields in the infinite-spin representations}

Starting from the posit that one-particles states look like one-particle states in every inertial frame, Wigner concluded that particles should be identified with unitary positive-energy representations of the (proper orthochronous) Poincaré group or its twofold covering. His famous classification [32] contains massive spin representations, massless helicity representations, and two one-parameter families of true resp. projective massless representations 
called "infinite spin" or "continuous spin". The parameter $\kappa^{2}>0$ is the eigenvalue of the Pauli-Lubanski operator $W^{2}=(P \wedge M)^{2}\left(P_{\rho}\right.$ is the momentum and $M_{\sigma \tau}$ are the Lorentz generators).

Weinberg [28-30] showed how one can associate local (or anti-local, in the projective case according to the spin-statistics theorem) quantum fields to all these representations except infinite spin. Let us from now on consider only the true (bosonic) representations.

In the massless case, only the field strengths can be constructed as local fields on the Fock space over the sum of Wigner representations with helicities $\pm h$ (which is irreducible when the parity is included). Their potentials necessarily violate either locality and covariance (e.g., in the Coulomb gauge) [28-30], or they must be constructed on an indefinite-metric Krein space of which the Fock space over the Wigner representation is a quotient. The latter option underlies all gauge-theoretic approaches to modern quantum field theory, with its introduction of more unphysical ghost fields to "compensate" states of negative probability.

For the irreducible infinite-spin representations, Yngvason [34] has shown a no-go theorem, that covariant local Wightman fields cannot exist. This was taken for a long time as a serious reason to consider these representations as "unphysical".

This conclusion is, however, a misunderstanding. From work of Buchholz and Fredenhagen [5] we know that quantum operators connecting scattering states with the vacuum may not in general be assumed to be localized in bounded spacetime regions. Instead, the best localization that can be proven is (a narrow "spacelike cone" arising by smearing) a "string" $S_{e}(x)=\{x+s e: s \geq 0\}\left(e^{2}=-1\right)$. So string-localization of interpolating fields may be a necessary feature of charged states in interacting theories, and only observables need to be point-localized. While the result in [5] applies to massive theories, it has a massless counterpart in the violation of Lorentz covariance of charged sectors of QED [9], due to the presence of "photon clouds" attached to charged fields that can - because of Gauß' law - at best be localized in a narrow cone.

String-localization surprisingly emerged in the context of infinite-spin representations, when Mund, Schroer and Yngvason [18], based on work by Brunetti, Guido and Longo [4], discovered a construction of fields $\varphi(e, x)$ transforming in the infinite-spin representation, that are localized along strings. Two such fields commute with each other whenever their strings $S_{e}(x)$ and $S_{e^{\prime}}\left(x^{\prime}\right)$ are spacelike separated.

The same authors also noticed that string-localized free fields may also be useful in the case of finite spin: e.g., one can construct a potential $A_{\mu}(e, x)$ of the Maxwell field strength $F_{\mu \nu}(x)$ directly on the ghost-free Fock space of the latter. This potential is manifestly presented as a string-integral over the field strength:

$$
A_{\mu}(e, x):=\int_{0}^{\infty} d s F_{\mu \nu}(x+s e) e^{\nu} .
$$

Similar constructions are possible for any mass and finite spin ([20] and [16] for the massless case, eq. (3.11) below and [16] for the massive case). Such potentials have the benefit $[22,23]$ that 
(i) in the massive case: they have an improved short-distance behaviour compared to the point-localized Proca potentials, which is a promising feature when the free fields are used perturbatively to set up an interacting theory; and unlike the latter, they admit a massless limit;

(ii) in the massless case: they are directly defined on the physical Hilbert space without the need to introduce Gupta-Bleuler conditions or compensating ghost fields.

These potentials were systematically studied for every integer spin $s$ in $[20]$, and in $[16,17]$ with the focus on their massless limit at fixed $s$, and on the discrepancy between the massless string-localized potentials and the massless limit of the massive string-localized potentials.

In contrast to the string-localized fields of finite spin like eq. (1.1), the infinite-spin fields of $[18,19]$ are "intrinsically string-localized": they cannot be expressed as stringintegrals over point-localized fields. This makes them quite non-trivial objects to study.

Also from different points of view, there is a lot of renewed interest in the infinitespin representations. Schuster and Toro [25, 26] and Rivelles [21] study quantum wave equations in a one-particle setting. Also their wave functions depend on an auxiliary fourvector $e$ which has, however, no direct geometric interpretation. "Localization" in the sense of causal commutators has no meaning in a one-particle (quantum-mechanical) approach. In [27], Schuster and Toro write down canonical commutation relations which are local both in $x$ and $e$, and it is not clear to the author how they realize such commutation relations in a Hilbert space - which would be at variance with Yngvason's no-go theorem.

Bekaert et al. [1-3] are pursuing a "Fierz-Pauli program" attempting to identify a classical action principle leading to wave equations compatible with the infinite-spin representation. With quantization beyond the scope of this program, the constraints due to Hilbert space positivity and causal commutation relations (addressed in, e.g., [16, 24]) play no role in their work.

In contrast, our work is placed in the setting of "Wigner quantization", where free fields are associated with a unitary Wigner representation by

$$
\phi_{M}(x)=\int d \mu_{0}(p) \sum_{n}\left[u_{M n}(p)(\vec{k}) a_{n}^{*}(p) e^{i p x}+v_{M n}(p) a_{n}(p, \vec{k}) e^{-i p x}\right]
$$

and the matrices $u$ and $v$ are "intertwiners" (see eq. (5.7)) between the unitary Wigner representation of the Lorentz group and the matrix representation (typically a tensor product of Lorentz matrices) under which the field transforms. They are needed to absorb the "Wigner rotations" that would otherwise spoil the covariant transformation law. For hermitean Bose fields, $v(p)=\overline{u(p)}$.

In this setting, Hilbert space positivity is manifest from the outset because eq. (1.2) is defined on the Fock space over the Wigner representation. Field equations, two-point functions and commutation relations follow without the need of a variational principle and "canonical" equal time commutation relations, i.e., they follow intrinsically (except for the choice of the localizing intertwiner functions) from Wigner representation theory. In this 
setting, the non-existence of a covariant quantum Maxwell potential on the physical Hilbert space is just the fact that the interwining relation has no solution.

This approach offers also an important new flexibility in perturbation theory [24]. While the Wigner representation fixes all properties of the particles, the choice of intertwiners determines (among other things) the short-distance behaviour of free fields that create these particles from the vacuum. Since UV singularities are a major problem of perturbation theory, one can benefit from the fact that string-localized fields have better UV properties and therefore admit renormalizable couplings that do not exist with pointlocalized fields (if the latter exist at all on a ghost-free Hilbert space). The case of massive vector bosons may be taken as an example: in order to control the UV problems of pointlocalized massive vector fields, the prevalent prescription treats them as massless fields in an indefinite-metric Hilbert space which "behave as if they were massive" thanks to the Higgs mechanism. In the string-localized approach, one may instead start with massive vector bosons and interpolating fields in their physical Hilbert space from the outset. (The Higgs field is still needed, but for a different reason, see below.)

In all cases of interactions mediated by string-localized fields, one has to observe that the obvious hazard of violating causality through the use of string-localized Lagrangeans in perturbation theory, can be controlled in terms of a certain cohomological "pair condition" [24] on the interaction terms: it secures the string-independence of the classical action, and is the first order condition for the string-independence of the perturbative quantum causal S-matrix. Higher order conditions may require additional "induced" interaction terms.

E.g., in the presence of selfinteracting massive vectormesons one needs an additional coupling of vectormesons to an Hermitian (Higgs) field in oder to uphold second order renormalizability. (Such compensations between fields with different spins have hitherto been expected to take place in the presence of supersymmetry; but whereas there are serious problems to maintain supersymmetry in second order, in the case of selfinteracting massive vector mesons this compensation is the very raison d'être for the Higgs particle without invoking a mechanism of spontaneous symmetry breaking, and without the need of unphysical ghost degrees of freedom.)

In the case of infinite-spin particles, the use of string-localized fields is not a choice (to improve the UV behaviour) but an intrinsic necessity [4]. Whether a pair condition can be fulfilled for any interaction with ordinary particles, is presently unknown. Schroer [24] discusses indications why this might not be the case (for infinite spin or already for some finite spin beyond a maximal value). As a consequence, these particles would be invisible in detectors ("inert"); the identification of a stress-energy tensor in the present work may be a starting point in order to investigate whether they might at least cause semiclassical gravitational back reactions.

The reader only interested in the infinite-spin stress-energy tensor, may jump directly to section 5, retaining from the preceeding sections only the properties Proposition 2.9 of the string-localized fields. These properties, although derived through the Pauli-Lubanski limit of finite mass and finite spin, refer directly to the Fock space over the infinite-spin representation, so that the construction of the stress-energy tensor is intrinsic. 


\subsection{Contents and plan of the paper}

Pauli-Lubanski limit. We study in section 4, how the string-localized infinite-spin fields of [18] are approximated by massive string-localized fields of finite spin, in the "PauliLubanski limit" $s \rightarrow \infty$ at fixed Pauli-Lubanski parameter $\kappa^{2}=m^{2} \cdot s(s+1)$. This limit is suggested by the fact that the Pauli-Lubanski operator $W^{2}=(P \wedge M)^{2}$ is a Casimir operator of the Lie algebra of the Poincaré group with eigenvalue $m^{2} \cdot s(s+1)$ in the representation $(m, s)$, and with eigenvalue $\kappa^{2}$ in the massless infinite-spin representation $U_{\kappa}^{1}$.

The Pauli-Lubanski limit is well known for the Wigner representations themselves [14], basically because the massless little group $\mathrm{E}(2)$ [32] is a contraction of the massive little group $\mathrm{SO}(3)$, see appendix $\mathrm{A}$. In the infinite-spin representations, the pseudo-translations (the subgroup $\mathbb{R}^{2} \subset \mathrm{E}(2)$ embedded into the proper orthochronous Lorentz group $\mathrm{SO}(1,3)_{+}^{\uparrow}$ ) are non-trivially represented with spectrum of their generators lying on a circle of radius $\kappa$. The corresponding basis of eigenfunctions $e^{i m \varphi}$ of the rotations of the little group ( $m \in \mathbb{Z}$ is the magnetic quantum number) can be approximated by the eigenfunctions $Y_{l m}$ $(l=s \rightarrow \infty,-l \leq m \leq l)$ of the finite-spin representations of $\mathrm{SO}(3)$, see appendix A.

But a "lift" of the limit of representations to the associated quantum fields is not known so far. An obvious obstruction seems to be that the (conserved and traceless) Proca potentials have a number of indices increasing with $s$, so that they are not even candidates for a "converging family of fields". Another obvious obstruction is that a limit of local commutator functions, if it exists, should be a local, and not a string-local commutator function that we know to be the best possible thing for infinite spin. But the most important obstruction is the singularity of the Proca potentials at $m=0$ which become stronger with increasing spin. It is related to the non-existence of point-localized currents and stressenergy tensors for the massless representations of finite helicity [31].

With string-localized massive potentials, these obstructions are absent. The potentials are manifestly string-localized from the beginning, they are regular at $m=0$, and they are neither traceless nor conserved, so that one may consider their divergences (called "escort fields") of fixed rank as natural candidates for converging families. Indeed this will turn out to be true, see section 4 .

This finding is a bit surprising. In [16], we have found that the "scalar escort field" converges in the massless limit at fixed $s$ to a true massless scalar field, while we are now claiming that in the Pauli-Lubanski limit, it converges to an infinite-spin field! Indeed, there is no contradiction. At finite mass, the scalar escort is coupled to the other escorts of any rank $r \leq s$ by field equations (eq. (3.12), eq. (3.15)), and each escort carries the entire spin $s$ representation. This coupling goes to zero in the massless limit at fixed $s$. But as our results implicitly show, it "remains stable" when $s$ increases at the same time, so that the limit field carries the entire infinite-spin representation.

Stress-energy tensor. In [16], we have constructed currents and stress-energy tensors for the massive finite spin representations that have a regular massless limit at fixed $s$. In the second part of our paper (section 5), we present a general construction that produces string-localized such densities also for the infinite-spin representations, and elucidate whether these exist as Wightman fields. In fact, this is expected not to be the case: their 
vacuum two-point functions are expected to diverge due to the infinitely many inner degrees of freedom that are summed over, and we give indications that this is indeed the case. While the vacuum two-point function is tedious to compute exactly, it is very easy to compute the thermal one-point function in KMS states. Here, the expected divergence [33] proportional to $2 s+1$ (in accord with the thermodynamical equipartition theorem) can be explicitly seen.

On the other hand, the commutator of the densities with the fields is a derivation that integrates to the infinitesimal gauge or Poincaré transformations. Because the latter are meaningful also at infinite spin, we expect the limit of the densities to exist at least "as derivations" on the algebra of fields.

Studying the existence and properties of currents and stress-energy tensors for the infinite-spin representations is of great interest, because even a classical Langrangean from which these could possibly be derived, is not known (see the "Fierz-Pauli program" of [3]). The intricacies of the quantum field theory, due to the conflict between Hilbert space positivity and causal point-localization, can only be overcome with string-localized fields.

In section 2, we review the essential features of string-localized infinite-spin fields and introduce the special fields that will appear in the stress-energy tensor. Section 3 prepares the ground for the Pauli-Lubanski limit. After these preparations, the initial main result of section 4, Proposition 4.3, which entails everything else, is very quickly obtained.

\section{String-localized infinite-spin fields}

The authors of $[18,19]$ constructed string-localized fields $\phi(e, x)$ on the Fock space over the infinite-spin representation $U_{\kappa}^{1}$ that transform like

$$
U_{\kappa}(a, \Lambda) \phi(e, x) U_{\kappa}(a, \Lambda)^{*}=\phi(\Lambda e, \Lambda x+a) .
$$

The action of $U_{\kappa}^{1}(a, \Lambda)$ on the one-particle space is constructed, as in Wigner's original approach [32], by induction from a representation $d_{\kappa}$ of the stabilizer group $\mathrm{E}(2)$ of the reference four-momentum $p_{0}=(1,0,0,1)^{t}$, and a family of Lorentz transformations $B_{p}$ for every $p \in H_{0}=\left\{p \in \mathbb{R}^{4}: p^{2}=0, p^{0}>0\right\}$, such that $B_{p} p_{0}=p$. The representation space $\mathcal{H}_{\kappa}=L^{2}\left(\kappa S^{1}\right)$ of $d_{\kappa}$ are the square-integrable functions of a two-dimensional vector $\vec{k}$, $\vec{k}^{2}=\kappa^{2}$. On such functions, the rotations and pseudo-translations act like

$$
\left(d_{\kappa}\left(R_{\alpha}\right) f\right)(\vec{k})=f\left(R_{-\alpha} \vec{k}\right), \quad\left(d_{\kappa}\left(T_{\vec{a}}\right) f\right)(\vec{k})=e^{i \vec{a} \cdot \vec{k}} f(\vec{k}) .
$$

The irreducible one-particle representation $U_{\kappa}^{1}$ induced from $d_{\kappa}$ is defined on squareintegrable functions on the zero mass shell $H_{0}$ with values in $\mathcal{H}_{\kappa}$, see appendix A. It immediately lifts to the representation $U_{\kappa}$ on the Fock space. The construction of hermitean fields out of creation and annihilation operators $a(p, \vec{k}), a^{*}(p, \vec{k})\left(p \in H_{0}, \vec{k} \in \kappa S^{1}\right)$ then proceeds in terms of "intertwiners" $u(e, p)$ :

$$
\phi_{u}(e, x)=\int d \mu_{0}(p) \int d \mu_{\kappa}(\vec{k})\left[u(e, p)(\vec{k}) a^{*}(p, \vec{k}) e^{i p x}+\overline{u(e, p)(\vec{k})} a(p, \vec{k}) e^{-i p x}\right]
$$


The intertwiners are distributions in $p$ and $e$ with values in $\mathcal{H}_{\kappa}$, that satisfy

$$
u(\Lambda e, \Lambda p)=d_{\kappa}\left(W_{\Lambda, p}\right) u(e, p) \quad\left(\Lambda \in \mathrm{SO}(1,3)_{+}^{\uparrow}\right)
$$

where $W_{\Lambda, p}=B_{\Lambda p}^{-1} \Lambda B_{p} \in \mathrm{E}(2)$ is the Wigner "rotation". ${ }^{1}$ This property ensures the transformation law eq. (2.1). In order to ensure that the commutator function vanishes for spacelike separated strings, it is crucial ([18], Theorem 3.3) that $u(e, p)$ is analytic in the $e$ variable in the complex tube $\mathcal{T}_{+}=\left\{e \in \mathbb{C}^{4}: e^{2}=-1\right.$, Im $\left.e \in V_{+}\right\}$, and satisfies certain local bounds in the tube as specified in ([18], Definition 3.1). The analyticity is necessary to ensure locality by a contour deformation argument. The bounds ensure that the boundary value of the analytic function $u$ at $\operatorname{Im} e \rightarrow 0$ defines an operator-valued distribution.

The intertwiner condition eq. (2.4) is equivalent to the pair of relations

$$
u(e, p)=u\left(B_{p}^{-1} e, p_{0}\right) \quad\left(p \in H_{0}\right)
$$

(which determines $u(e, p)$ for all $p \in H_{0}$ as soon as $u_{0}(e)=u\left(e, p_{0}\right)$ is given), and

$$
u_{0}(W e)=d_{\kappa}(W) u_{0}(e) \quad(W \in \mathrm{E}(2))
$$

which determines $u_{0}(e)$ along each orbit of $e$ under the little group. The argument is standard: let $\xi_{0}=\frac{1}{2}(1,0,0,-1)^{t}$ and $\xi(\vec{a}):=T_{\vec{a}} \xi_{0}=\frac{1}{2}\left(\vec{a}^{2}+1,2 a^{1}, 2 a^{2}, \vec{a}^{2}-1\right)^{t}$. The orbits of $\mathrm{E}(2)$ are parametrized by the invariants $e^{2}$ and $\left(e p_{0}\right)$, and $e$ along each orbit with $\left(e p_{0}\right) \neq 0$ is parametrized by $e(\vec{a})=\frac{e^{2}}{2\left(e p_{0}\right)} p_{0}+\left(e p_{0}\right) \xi(\vec{a}), \vec{a} \in \mathbb{R}^{2}$. Because $e_{0}=e(\overrightarrow{0})$ is fixed by the rotations of $\mathrm{E}(2), u_{0}\left(e_{0}\right)(\vec{k})$ is a constant function of $\vec{k} \in \kappa S^{1}$ by the first of eq. (2.2). Its value $f\left(e^{2},\left(e p_{0}\right)\right)$ is an arbitrary function of the orbit. The translations of $\mathrm{E}(2)$ then determine

$$
u_{0}(e)(\vec{k})=f\left(e^{2},\left(e p_{0}\right)\right) \cdot e^{-i \frac{(e E(\vec{k}))}{\left(e p_{0}\right)}}
$$

everywhere along the orbit, by the second of eq. (2.2). Here, $E: \mathbb{R}^{2} \rightarrow \mathbb{R}^{4}$ is the standard embedding $E(\vec{k}):=\left(0, k^{1}, k^{2}, 0\right)^{t}$ into Minkowski space. The function $f(x, y)$ is not determined by eq. (2.6). By eq. (2.5), one gets

$$
u_{f}(e, p)(\vec{k})=f\left(e^{2},(e p)\right) \cdot e^{-i \frac{\left(e E_{p}(\vec{k})\right)}{(e p)}}
$$

where $E_{p}(\vec{k}):=B_{p} E(\vec{k})$.

These are identical with the "smooth" solutions to the three differential equations (3.6)-(3.8) in [25], that are the infinitesimal version of eq. (2.6), lifted to $p \in H_{0}$ by eq. (2.5). The "singular" solutions supported on the orbits with $(e p)=0$ are not admissible as intertwiners.

\footnotetext{
${ }^{1}$ Of course, it is not a rotation, nor is $B_{p}$. We sloppily adopt the terminology Wigner "rotation" and standard "boost" for $B_{p}: p_{0} \mapsto p$ from the massive case.
} 


\subsection{The scalar standard field}

Analyticity in the forward tube $\mathcal{T}_{+}$of $e$ requires to take $1 /(e p)$ in eq. (2.7) as the distribution $1 /(e p)_{+}$, because $(e p) \in \mathbb{C}_{+}$if $e \in \mathcal{T}_{+}$. The simplest solution

$$
\widetilde{u}^{\kappa}(e, p)(\vec{k}):=e^{-i \frac{\left(e E_{p}(\vec{k})\right)}{(e p)_{+}}}
$$

solves the wave equations eq. (3.17)-(3.18) in [25] along with their subsidiary conditions eq. (3.19)-(3.20) with $n=0$. But it does not satisfy the bounds required to prove the vanishing of commutators when the strings are spacelike separated ([16], Theorem 3.3). E.g., for $p=(\mu, 0,0, \mu)^{t}$ and $e=\left(i x, \sqrt{1-x^{2}}, 0,0\right)^{t} \in \mathcal{T}_{+}$, the estimate $\left\|\widetilde{u}^{\kappa}(e, p)\right\| \geq$ $\exp \left(\frac{\sqrt{1-x^{2}}}{2 x} \frac{\kappa}{\mu}\right)$ violates every power law bound as $x \rightarrow 0$ and $\mu \rightarrow 0$. Instead:

Proposition 2.1 (see also [12], pp. 42ff and Remark after [19], Definition 3.1) In the tube $\mathcal{T}_{+}$, it holds $\left|e^{-i \frac{\left(e E_{p}(\vec{k})\right)}{(e p)}}\right| \leq\left|e^{\frac{i \kappa}{(e p)}}\right|$. Therefore, the solution

$$
u^{\kappa(0)}(e, p)(\vec{k})=e^{-i \kappa \frac{\sqrt{-e^{2}}}{(e p)+}} \cdot e^{-i \frac{\left(e E_{p}(\vec{k})\right)}{(e p)_{+}}}
$$

at real e exists as a weakly continuous $L^{2}\left(\kappa S^{1}\right)$-valued function. It is the intertwiner of a string-localized field $\phi^{\kappa(0)}(e, x)$.

Remark 2.2 Although we always put $e^{2}=-1$, we write $\sqrt{-e^{2}}$ because we are going to take derivatives w.r.t. e by defining intertwiners as homogeneous functions $u(\lambda e):=u(e)(\lambda>0)$ for all spacelike e. In the sequel, we shall refer to eq. (2.9) as the "standard intertwiner", and the associated field $\phi^{\kappa(0)}$ as the "standard (string-localized infinite-spin) field". We call $\omega^{\kappa}(e, p)=e^{-i \kappa \frac{\sqrt{-e^{2}}}{(e p)+}}$ the "Köhler factor" [12]. It appears only in the combination eq. (2.9) where it cancels the essential singularity of eq. (2.8).

Proof of Proposition 2.1. If $e=e^{\prime}+i e^{\prime \prime}$ is in the tube, then $e^{\prime \prime} \in V_{+}$and $\left(e^{\prime} e^{\prime \prime}\right)=0$, hence $e^{\prime}$ is spacelike (or $=0$ ). $e^{2}=-1$ implies $-1<e^{\prime 2} \leq 0$ and $0<e^{\prime \prime 2} \leq 1$.

For $e \in \mathcal{T}_{+}$, the real part of the exponent $-i \frac{\left(e E_{p}(\vec{k})\right)}{(e p)}$ is

$$
\frac{\left(e^{\prime \prime} E_{p}(\vec{k})\right)\left(e^{\prime} p\right)-\left(e^{\prime} E_{p}(\vec{k})\right)\left(e^{\prime \prime} p\right)}{|(e p)|^{2}}=\frac{\left(f^{\prime \prime} E(\vec{k})\right)\left(f^{\prime} p_{0}\right)-\left(f^{\prime} E(\vec{k})\right)\left(f^{\prime \prime} p_{0}\right)}{\left|\left(f p_{0}\right)\right|^{2}}
$$

where $f:=B_{p}^{-1} e=f^{\prime}+i f^{\prime \prime},\left(f^{\prime} f^{\prime \prime}\right)=0, f^{\prime \prime} \in V_{+}, f^{\prime 2}-f^{\prime \prime 2}=-1$. Parametrize $f^{\prime}=$ $\alpha^{\prime} p_{0}+\beta^{\prime} \xi_{0}+E\left(\overrightarrow{f^{\prime}}\right), f^{\prime \prime}=\alpha^{\prime \prime} p_{0}+\beta^{\prime \prime} \xi_{0}+E\left(\vec{f}^{\prime \prime}\right)$, such that $\left(\overrightarrow{f^{\prime}} \vec{f}^{\prime \prime}\right)=\alpha^{\prime} \beta^{\prime \prime}+\alpha^{\prime \prime} \beta^{\prime}$, and $\beta^{\prime \prime}>0$, $\vec{f}^{\prime \prime 2}<2 \alpha^{\prime \prime} \beta^{\prime \prime}$, and $2 \alpha^{\prime} \beta^{\prime}-\vec{f}^{\prime 2}-2 \alpha^{\prime \prime} \beta^{\prime \prime}+\vec{f}^{\prime \prime 2}=-1$, hence $\vec{f}^{\prime 2}<2 \alpha^{\prime} \beta^{\prime}+1$.

Then, the numerator is (cf. [10])

$$
\begin{aligned}
\left(\beta^{\prime \prime} \vec{f}^{\prime}-\beta^{\prime} \vec{f}^{\prime \prime}\right) \cdot \vec{k} & \leq \kappa\left|\beta^{\prime \prime} \vec{f}^{\prime}-\beta^{\prime} \vec{f}^{\prime \prime}\right|=\kappa \sqrt{\beta^{\prime \prime 2} \vec{f}^{\prime 2}+\beta^{\prime 2} \vec{f}^{\prime \prime 2}-2 \beta^{\prime} \beta^{\prime \prime}\left(\overrightarrow{f^{\prime}} \overrightarrow{f^{\prime \prime}}\right)} \leq \\
& \leq \kappa \sqrt{\beta^{\prime \prime 2}\left(2 \alpha^{\prime} \beta^{\prime}+1\right)+\beta^{\prime 2} 2 \alpha^{\prime \prime} \beta^{\prime \prime}-2 \beta^{\prime} \beta^{\prime \prime}\left(\alpha^{\prime} \beta^{\prime \prime}+\alpha^{\prime \prime} \beta^{\prime}\right)}=\kappa \beta^{\prime \prime} .
\end{aligned}
$$

Thus $\left|e^{-i \frac{\left(e E_{p}(\vec{k})\right)}{(e p)}}\right| \leq e^{\kappa \frac{\beta^{\prime \prime}}{\left(e p_{0}\right)^{2}}}=\left|\omega^{\kappa}(e, p)^{-1}\right|$. It follows that $\omega^{\kappa}(e, p) \cdot e^{-i \frac{\left(e E_{p}(\vec{k})\right)}{(e p)}}$ is an analytic function in $\mathcal{T}_{+}$bounded by 1 . Thus it satisfies the bounds specified in ([16], Definition 3.1) 
and the remark following it, hence its boundary value eq. (2.9) is well-defined as a function, and defines a string-localized field by ([16], Theorem 3.3).

Mund, Schroer and Yngvason in [19] also gave solutions to the intertwiner relation eq. (2.4) in a different form (for $e^{2}=-1$ ):

$$
U_{F}(e, p)(\vec{k})=\int d^{2} a e^{i \vec{k} \cdot \vec{a}} F\left(\left(e B_{p} \xi(\vec{a})\right)\right) .
$$

The function $F(z)$ must be analytic and polynomially bounded in the upper half-plane, hence its Fourier transform $\widehat{F}$ is supported on $\mathbb{R}_{+}$. One can bring this form into the form eq. (2.7): with the Fourier representation of $F(z)$, the $\vec{a}$-integration becomes Gaussian and can be performed when $e \in \mathcal{T}_{+}$, with the result (see [10])

$$
U_{F}(e, p)(\vec{k})=u^{\kappa(0)}(e, p)(\vec{k}) \cdot\left[\frac{2 \pi i}{(e p)_{+}} \int_{0}^{\infty} \frac{d t}{t} \widehat{F}\left(\frac{\kappa}{\sqrt{-e^{2}}} t\right) e^{-i \frac{\kappa \sqrt{-e^{2}}}{(e p)_{+}} \frac{(t-1)^{2}}{2 t}}\right] .
$$

The choice $2 \pi \widehat{F}\left(\frac{\kappa}{\sqrt{-e^{2}}} t\right)=\delta(t-1)$ gives the intertwiner $U_{F}=\frac{i}{(e p)_{+}} u^{\kappa(0)}$, i.e., the standard field is $\phi^{\kappa(0)}(e, x)=-\left(e \partial_{x}\right) \phi_{F}(e, x)$.

We introduce the string-integration operator

$$
\left(I_{e} X\right)(x):=\int_{0}^{\infty} d s X(x+s e)
$$

already occurring in eq. (1.1). If $X(e, x)$ is localized along the string $e$, then so is $I_{e} X(e, x)$. One has

$$
\left(e \partial_{x}\right) I_{e} X=I_{e}\left(e \partial_{x}\right) X=-X .
$$

In momentum space, acting on $e^{i p x}$, this is the multiplication operator by

$$
I_{e}(p)=\frac{i}{(e p)_{+}} \equiv \lim _{\varepsilon \searrow 0} \frac{i}{(e p)+i \varepsilon}
$$

as a distribution. We also introduce

$$
\left(J_{e}\right)_{\mu}^{\nu}:=I_{e}\left[e^{\nu} \partial_{\mu}-\delta_{\mu}^{\nu}\left(e \partial_{\nu}\right)\right]=\delta_{\mu}^{\nu}+I_{e} e^{\nu} \partial_{\mu},
$$

because of eq. (2.13). In momentum space, this is the multiplication operator by

$$
J_{e}(p)_{\mu}{ }^{\nu}=(e p) \partial_{e^{\mu}} \frac{e^{\nu}}{(e p)_{+}}=\delta_{\mu}^{\nu}-p_{\mu} \frac{e^{\nu}}{(e p)_{+}},
$$

so that

$$
(e p) \partial_{e^{\mu}} \widetilde{u}^{\kappa}(e, p)(\vec{k})=-i\left(J_{e}(p) E_{p}(\vec{k})\right)_{\mu} \cdot \widetilde{u}^{\kappa}(e, p)(\vec{k}) .
$$

Varying w.r.t. $e$, one has

$$
(e p) \partial_{e^{\lambda}} J_{e}(p)_{\mu}{ }^{\nu}=-p_{\mu} J_{e}(p)_{\lambda}^{\nu}
$$


Because we are going to take derivatives w.r.t. $e$ of intertwiners multiplied with the Köhler factor, it is convenient to introduce

$$
D_{e}(p):=\partial_{e}-\frac{i \kappa}{\sqrt{-e^{2}}} \frac{J_{e}(p) e}{(e p)_{+}},
$$

so that $D_{e}(p)\left(\omega^{\kappa} \widetilde{u}(e, p)\right)=\omega^{\kappa} \partial_{e} \widetilde{u}(e, p)$. Acting on the corresponding fields, this is the operator

$$
D_{e}=\partial_{e}-\frac{\kappa}{\sqrt{-e^{2}}} I_{e} J_{e} e
$$

Proposition 2.3 The standard string-localized infinite-spin field $\phi^{\kappa(0)}(e, x)$ satisfies the equations of motion

$$
\square_{x} \phi^{\kappa(0)}=\left(e \partial_{e}\right) \phi^{\kappa(0)}=\left(\partial_{x} D_{e}\right) \phi^{\kappa(0)}=\left(\left(e \partial_{x}\right)^{2} D_{e}^{2}+\kappa^{2}\right) \phi^{\kappa(0)}=0 .
$$

The last two equations in eq. (2.21) are equivalent to

$$
\sqrt{-e^{2}}\left(\partial_{x} \partial_{e}\right) \phi^{\kappa(0)}=\sqrt{-e^{2}}\left(e \partial_{x}\right) \square_{e} \phi^{\kappa(0)}=-\kappa \phi^{\kappa(0)} .
$$

The Pauli-Lubanski equation follows:

$$
W^{2} \phi^{\kappa(0)}=\kappa^{2} \phi^{\kappa(0)}
$$

where $W^{2}=\square_{x}\left(e^{2} \square_{e}-\left(e \partial_{e}\right)^{2}-\left(e \partial_{e}\right)\right)+2\left(e \partial_{x}\right)\left(\partial_{x} \partial_{e}\right)\left(e \partial_{e}\right)-e^{2}\left(\partial_{x} \partial_{e}\right)^{2}-\left(e \partial_{x}\right)^{2} \square_{e}$ is the Pauli-Lubanski operator.

Proof. The Klein-Gordon equation is fulfilled by construction, and the homogeneity in $e$ is manifest from eq. (2.9). Using eq. (2.17) and $p^{2}=\left(p E_{p}(\vec{k})\right)=0$, hence $\left(p J_{e} E_{p}(\vec{k})\right)=0$ and $\left(J_{e} E_{p}(\vec{k})\right)^{2}=\left(E_{p}(\vec{k})\right)^{2}=-\kappa^{2}$, one computes

$$
\left(p \partial_{e}\right) \widetilde{u}^{\kappa}(e, p)=0, \quad\left((e p)^{2} \square_{e}-\kappa^{2}\right) \widetilde{u}^{\kappa}(e, p)=0,
$$

hence

$$
\left(p D_{e}\right) u^{\kappa(0)}(e, p)=0, \quad\left((e p)^{2} D_{e}^{2}-\kappa^{2}\right) u^{\kappa(0)}(e, p)=0 .
$$

These relations for the intertwiner are equivalent to the last two equations in eq. (2.21). The first of eq. (2.22) is equivalent to the third in eq. (2.21) by eq. (2.13), and the second of eq. (2.22) follows from the last of eq. (2.21) by a lengthy calculation using also the second and third of eq. (2.21). Eq. (2.23) is then a consequence of the previous.

\section{$2.2 \quad$ Tensor fields I}

We shall later also need tensor fields that transform like

$$
U_{\kappa}(a, \Lambda) \phi_{\mu_{1} \ldots \mu_{r}}(e, x) U_{\kappa}(a, \Lambda)^{*}=\prod \Lambda_{\mu_{i}}^{\nu_{i}} \phi_{\nu_{1} \ldots \nu_{r}}(\Lambda e, \Lambda x+a) .
$$


They are formed with intertwiners that, regarded as functions with values in $\left(\mathbb{R}^{4}\right)^{\otimes r} \otimes \mathcal{H}_{\kappa}$, satisfy

$$
u(\Lambda e, \Lambda p)=\left(\left(\Lambda^{\otimes r}\right) \otimes d_{\kappa}\left(W_{\Lambda, p}\right)\right) u(e, p) .
$$

To simplify notation, we write contractions as $a^{\mu} b_{\mu} \equiv(a b) \equiv a^{t} b$, and for symmetric tensors of rank $r$, we write

$$
X(v) \equiv v^{\mu_{1}} \ldots v^{\mu_{r}} X_{\mu_{1} \ldots \mu_{r}} \equiv v^{t \otimes r} X \quad\left(v \in \mathbb{R}^{4}\right) .
$$

The tensor components are recovered by differentiation w.r.t. $v^{\mu}$.

Definition 2.4 We introduce the symmetric rank $r$ tensor intertwiners

$$
u^{\kappa(r)}(e, p)(\vec{k}):=(-\kappa)^{-r}\left(J_{e}(p) E_{p}(\vec{k})\right)^{\otimes r} \cdot u^{\kappa(0)}(e, p)(\vec{k}),
$$

where $E_{p}: \mathbb{R}^{2} \rightarrow \mathbb{R}^{4}$ is the anti-isometric embedding $\left(E_{p}(\vec{k})^{2}=-\vec{k}^{2}\right)$ introduced in eq. (2.7). The associated tensor fields are called $\phi^{\kappa(r)}(e)$.

Proposition 2.5 The symmetric tensor fields $\phi^{\kappa(r)}$ are recursively related to $\phi^{\kappa(0)}$ by

$$
-\kappa \phi^{\kappa(r+1)}(e, x, v)=\left(r \cdot\left(v \partial_{x}\right)+\left(e \partial_{x}\right)\left(v D_{e}\right)\right) \phi^{\kappa(r)}(e, x, v) .
$$

Proof. Eq. (2.17) and $\left(r \cdot p_{\mu}+(e p) \partial_{e^{\mu}}\right) \circ J_{e}^{\otimes r}=J_{e}^{\otimes r}(e p) \circ \partial_{e^{\mu}}$ imply

$$
-i\left(v^{t} J_{e}(p) E_{p}(\vec{k})\right)^{r+1} \cdot \widetilde{u}^{\kappa}(e, p)(\vec{k})=\left(r \cdot(v p)+(e p)\left(v \partial_{e}\right)\right)\left(v^{t} J_{e}(p) E_{p}(\vec{k})\right)^{r} \cdot \widetilde{u}^{\kappa}(e, p)(\vec{k}) .
$$

Restoring the Köhler factor, we have to replace $\partial_{e}$ by $D_{e}(p)$ defined in eq. (2.19):

$$
i \kappa u^{\kappa(r+1)}(e, p, v)(\vec{k})=\left(r \cdot(v p)+(e p)\left(v D_{e}(p)\right)\right) u^{\kappa(r)}(e, p, v)(\vec{k}) .
$$

This is the momentum space version of eq. (2.27).

We may take eq. (2.27) as recursive definitions. The covariance of eq. (2.27) and eq. (2.28) ensures that $\phi^{\kappa(r)}$ and their intertwiners $u^{\kappa(r)}$ transform according to eq. (2.24) and eq. (2.25), respectively, and the bounds of ([16], Definition 3.1) are inherited from the bound of $u^{\kappa(0)}$ because $\left(J_{e}(p) E_{p}(\vec{k})\right)^{2}=-\kappa^{2}$.

Corollary 2.6 $\phi_{\mu_{1} \ldots \mu_{r}}^{\kappa(r)}(e, x)$ are string-localized tensor fields.

\subsection{Tensor fields II}

Definition 2.7 We introduce a second family of symmetric rank $r$ tensor fields

$$
\Phi^{\kappa(r)}(e, x)=2^{r / 2} \cdot\left(S_{r} \sum_{2 k \leq r} \gamma_{k}^{r} \cdot\left(J_{e}^{\otimes 2} \eta \circ \eta^{t}\right)^{\otimes k}\right) \phi^{\kappa(r)}(e, x)
$$

with the coefficients $\gamma_{k}^{r}=\frac{1}{4^{k} k !} \frac{r !}{(r-2 k) !} \frac{1}{(1-r)_{k}}$, and $S_{r}$ the projection onto the symmetric tensors in $\left(\mathbb{R}^{4}\right)^{\otimes r}$. The associated string-localized tensor fields are called $\Phi^{\kappa(r)}(e) . J_{e}$ is the operator given in eq. (2.15). (Trivially, $\Phi^{\kappa(0)}=\phi^{\kappa(0)}$ and $\Phi^{\kappa(1)}=\sqrt{2} \cdot \phi^{\kappa(1)}$.) 
Remark 2.8 The operations on $\phi^{\kappa(r)}$ that define $\Phi^{\kappa(r)}$ preserve the localization and covariance eq. (2.24). Thus, the latter are again string-localized tensor fields.

The following proposition exhibits the advantage of the new fields $\Phi^{\kappa(r)}$, that will become important in section 5 .

Proposition 2.9 (i) The intertwiner of $\Phi^{\kappa(r)}$ equals

$$
U^{\kappa(r)}(e, p)(\vec{k})=\frac{2^{r / 2}}{(-\kappa)^{r}} \cdot\left(J_{e}(p) E_{p}\right)^{\otimes r}\left(\Pi_{2}^{(r)}\left(\vec{k}^{\otimes r}\right)\right) \cdot u^{\kappa(0)}(e, p)(\vec{k}) .
$$

Here, $\Pi_{2}^{(r)}$ is the projection (of two-dimensional range) onto the symmetric traceless tensors in $\left(\mathbb{R}^{2}\right)^{\otimes r}$.

(ii) For $\vec{k}=\kappa\left(\begin{array}{c}\cos \varphi \\ \sin \varphi\end{array}\right)$ and $\varepsilon_{ \pm}=\frac{1}{\sqrt{2}}\left(\begin{array}{c}1 \\ \pm i\end{array}\right)$, one has the decomposition into helicity eigenstates

$$
\frac{2^{r / 2}}{\kappa^{r}} \Pi_{2}^{(r)}\left(\vec{k}^{\otimes r}\right)= \begin{cases}1 & (r=0) \\ e^{-i r \varphi} \varepsilon_{+}^{\otimes r}+e^{i r \varphi} \varepsilon_{-}^{\otimes r} & (r>0),\end{cases}
$$

hence $U^{\kappa(0)}=u^{\kappa(0)}$, and for $r>0$

$$
U^{\kappa(r)}(e, p)(\vec{k})=(-1)^{r}\left(e^{-i r \varphi} \mathcal{E}_{+}(e, p)^{\otimes r}+e^{i r \varphi} \mathcal{E}_{-}(e, p)^{\otimes r}\right) \cdot u^{\kappa(0)}(e, p)(\vec{k}),
$$

where $\mathcal{E}_{ \pm}(e, p):=J_{e}(p) E_{p} \varepsilon_{ \pm}$.

Remark 2.10 The presence of the factor $u^{\kappa(0)}(\vec{k})$ prevents the interpretation of $\Phi^{\kappa(r)}(e)$ as "fields of sharp helicity", in accord with the irreducibility of the infinite-spin representation.

Proof of Proposition 2.9. By eq. (2.29), the intertwiner of $\Phi^{\kappa(r)}$ is

$$
U^{\kappa(r)}(e, p)(\vec{k})=2^{r / 2} \cdot\left(S_{r} \sum_{2 k \leq r} \gamma_{k}^{r} \cdot\left(J_{e}(p)^{\otimes 2} \eta \circ \eta^{t}\right)^{\otimes k}\right) u^{\kappa(r)}(e, p)(\vec{k})
$$

with $J_{e}(p)$ given in eq. (2.16). By eq. (2.26), the intertwiner $u^{\kappa(r)}$ is in the range of the operator $\left(J_{e}(p) E_{p}\right)^{\otimes r}$. Therefore, we may consider the operators

$$
J_{e}(p)^{\otimes 2} \eta \circ \eta^{t}\left(J_{e}(p) E_{p}\right)^{\otimes 2}:\left(\mathbb{R}^{2}\right)^{\otimes 2} \rightarrow\left(\mathbb{R}^{4}\right)^{\otimes 2}
$$

appearing in eq. (2.33) when eq. (2.26) is inserted. Using the standard vectors $p_{0}=$ $(1,0,0,1)^{t}$ and $\xi_{0}=\left(\frac{1}{2}, 0,0,-\frac{1}{2}\right)^{t}$, we have $\eta=-E^{\otimes 2} \delta_{2}+p_{0} \otimes \xi_{0}+\xi_{0} \otimes p_{0}$ as tensors in $\left(\mathbb{R}^{4}\right)^{\otimes 2}$, where $\delta_{2}=\vec{e}_{1} \otimes \vec{e}_{1}+\vec{e}_{2} \otimes \vec{e}_{2}$ as a tensor in $\left(\mathbb{R}^{4}\right)^{\otimes 2}$. Applying the standard "boost" $B_{p}: p_{0} \mapsto p$, we get

$$
\eta=-E^{\otimes 2} \delta_{2}+p \otimes B_{p} \xi_{0}+B_{p} \xi_{0} \otimes p .
$$

Because $p^{2}=0$, we have $\eta^{t} J_{e}(p)^{\otimes 2}=\eta^{t}-\left(B_{p} \xi_{0}\right)^{t} \otimes p^{t}-p^{t} \otimes\left(B_{p} \xi_{0}\right)^{t}$, hence

$$
J_{e}(p)^{\otimes 2} \eta=\left(J_{e}(p) E_{p}\right)^{\otimes 2} \delta_{2}, \quad \eta^{t}\left(J_{e}(p) E_{p}\right)^{\otimes 2}=\eta^{t} E_{p}^{\otimes 2}=\delta_{2}^{t} E_{p}^{t \otimes 2} E_{p}^{\otimes 2}=\delta_{2}^{t},
$$


because $p$ is orthogonal to the range of $E_{p}$. Thus, eq. (2.33) can be rewritten as

$$
U^{\kappa(r)}(e, p)(\vec{k})=\frac{2^{r / 2}}{\kappa^{r}} \cdot\left(J_{e}(p) E_{p}\right)^{\otimes r}\left(S_{r} \sum_{2 k \leq r} \gamma_{k}^{r} \cdot\left(\delta_{2} \delta_{2}^{t}\right)^{\otimes k} \otimes \mathbb{I}_{2}^{\otimes r-2 k}\right) \vec{k}^{\otimes r} \cdot u^{\kappa(0)}(e, p)(\vec{k}) .
$$

The operator in brackets is the projection $\Pi_{2}^{(r)}$ onto the symmetric traceless tensors in $\left(\mathbb{R}^{2}\right)^{\otimes r}$ (this is in fact the defining property of the coefficients $\gamma_{k}^{r}[11]$ ). This proves the claim (i). Now, write $\vec{k}=\frac{\kappa}{\sqrt{2}}\left(e^{-i \varphi} \varepsilon_{+}+e^{+i \varphi} \varepsilon_{-}\right)$. Then eq. (2.31) is a well-known identity (that may be proven by induction in $r$ ), and eq. (2.32) follows.

Proposition 2.11 Besides the massless Klein-Gordon equation, the infinite-spin symmetric tensor fields $\Phi_{\mu_{1} \ldots \mu_{r}}^{\kappa(r)}(e, x)$ satisfy the equations of motion and constraints

$$
\begin{aligned}
& \eta^{\mu \nu} \Phi_{\mu \nu \mu_{3} \ldots \mu_{r}}^{\kappa(r)}=0, \quad \partial^{\mu} \Phi_{\mu \mu_{2} \ldots \mu_{r}}^{\kappa(r)}=0, \quad e^{\mu} \Phi_{\mu \mu_{2} \ldots \mu_{r}}^{\kappa(r)}=0, \\
& \left(e \partial_{e}\right) \Phi_{\mu_{1} \ldots \mu_{r}}^{\kappa(r)}=0, \quad\left(\partial_{x} D_{e}\right) \Phi_{\mu_{1} \ldots \mu_{r}}^{\kappa(r)}=0,
\end{aligned}
$$

as well as the coupling relations for $r \geq 2$

$$
\begin{aligned}
& \left(\left(e \partial_{x}\right) D_{e^{\mu_{1}}} \Phi_{\mu_{2} \ldots \mu_{r+1}}^{\kappa(r)}+\sum_{i=2}^{r+1} \partial_{\mu_{i}} \Phi_{\mu_{1} \ldots \mu_{i-1} \mu_{i+1} \ldots \mu_{r+1}}^{\kappa(r)}\right)+\left(\mu_{1} \leftrightarrow \mu_{2}\right)= \\
& \quad=-\sqrt{2} \kappa\left(\Phi_{\mu_{1} \ldots \mu_{r+1}}^{\kappa(r+1)}-\frac{1}{2}\left(E_{e}\right)_{\mu_{1} \mu_{2}} \Phi_{\mu_{3} \ldots \mu_{r+1}}^{\kappa(r-1)}\right) .
\end{aligned}
$$

Here, $\left(E_{e}\right)_{\mu \nu}$ is the integral- and differential operator $\left(\left(J_{e} \otimes J_{e}\right) \eta\right)_{\mu \nu}$. For $r=0$, the second term in the bracket on the r.h.s. is absent, and for $r=1$ is replaced by $-\left(E_{e}\right)_{\mu_{1} \mu_{2}} \Phi^{\kappa(0)}(v)$.

Proof. We proceed in momentum space, where $\partial_{x}=i p$ on the intertwiners eq. (2.32). Eq. (2.34) and eq. (2.35) follow by a direct computation, using $\left(\mathcal{E}_{ \pm} \mathcal{E}_{ \pm}\right)=0$ and $\left(p \mathcal{E}_{ \pm}\right)=$ $\left(e \mathcal{E}_{ \pm}\right)=\left(p \partial_{e}\right) \mathcal{E}_{ \pm}=0$, as well as $D_{e} u^{\kappa(0)}=\omega^{\kappa} \partial_{e} \widetilde{u}^{\kappa}$. For the coupling relations, apply $i(e p) D_{e^{\mu_{1}}}$ to $U_{\mu_{2} \ldots \mu_{r+1}}^{\kappa(r)}$. Use $\partial_{e^{\mu}} \mathcal{E}_{ \pm \nu}=-p_{\nu} \mathcal{E}_{ \pm \mu}$ by eq. (2.18), which cancels the spatial derivatives on the 1.h.s. of eq. (2.36), and $i(e p) D_{e^{\mu}} u^{\kappa(0)}=\left(J_{e}(p) E_{p}(\vec{k})\right)_{\mu} u^{\kappa(0)}=$ $\frac{\kappa}{\sqrt{2}}\left(e^{-i \varphi} \mathcal{E}_{+}+e^{i \varphi} \mathcal{E}_{-}\right) u^{\kappa(0)}$ by eq. (2.17). This produces the term $\left(\mathrm{id}+\pi_{12}\right)\left(\widetilde{\mathcal{E}}_{+}+\widetilde{\mathcal{E}}_{-}\right) \otimes$ $\left(\widetilde{\mathcal{E}}_{+}^{\otimes r}+\widetilde{\mathcal{E}}_{-}^{\otimes r}\right)$ where $\widetilde{\mathcal{E}}_{ \pm} \equiv e^{\mp i \varphi} \mathcal{E}_{ \pm}$and $\pi_{12}$ is the permutation of the first two tensor factors. This tensor trivially equals

$$
=2\left(\widetilde{\mathcal{E}}_{+}^{\otimes r+1}+\widetilde{\mathcal{E}}_{-}^{\otimes r+1}\right)+\left(\mathcal{E}_{+} \otimes \mathcal{E}_{-}+\mathcal{E}_{-} \otimes \mathcal{E}_{+}\right) \otimes\left(\widetilde{\mathcal{E}}_{+}^{\otimes r-1}+\widetilde{\mathcal{E}}_{-}^{\otimes r-1}\right) .
$$

Finally, $\mathcal{E}_{+} \otimes \mathcal{E}_{-}+\mathcal{E}_{-} \otimes \mathcal{E}_{+}=\left(J_{e}(p) E_{p}\right)^{\otimes 2}\left(\delta_{2}\right)=-E_{e}(p)$, as in the proof of Proposition 2.9, implies the claim.

\section{Preparations for the Pauli-Lubanski limit}

\subsection{Two-point functions and commutators: basics}

Our fields are free fields, so that the entire information resides in their two-point functions, which in particular determine the commutator functions. It is convenient to express the 
two-point functions as integral or differential operators acting on the canonical scalar twopoint function $(\Omega, \varphi(x) \varphi(y) \Omega)=\Delta_{m}(x-y)$. This amounts to the insertion of a "two-point kernel" into the Fourier representation:

$$
(\Omega, X(x) Y(y) \Omega)=\int d \mu_{m}(p) e^{-i p(x-y)} \cdot M_{m}^{X, Y}(p),
$$

where $d \mu_{m}(p)=(2 \pi)^{-3} d^{4} p \delta\left(p^{2}-m^{2}\right) \theta\left(p^{0}\right)$ is the Lorentz invariant measure on the positive energy mass shell $H_{m}$. E.g., the canonical scalar field and the Proca field have the two-point kernels

$$
M_{m}^{\varphi, \varphi}=1 \quad \text { resp. } \quad M_{m}^{A_{\mu}^{\mathrm{P}}, A_{\nu}^{\mathrm{P}}}(p)=-\pi_{\mu \nu} \equiv-\left(\eta_{\mu \nu}-\frac{p_{\mu} p_{\nu}}{m^{2}}\right) .
$$

\subsection{Two-point functions of string-localized spin 1 fields}

For the massive Proca field we introduce the string-localized potential by the same formula as for the Maxwell potential eq. (1.1); but in this case it can also be expressed in terms of the point-localized potential $A_{\nu}^{\mathrm{P}}=-\frac{1}{m^{2}} \partial^{\mu} F_{\mu \nu}^{\mathrm{P}}$ that exists on the Hilbert space:

$$
A_{\mu}(e, x):=\left(I_{e} F_{\mu \nu}^{\mathrm{P}}\right)(x) e^{\nu}=A_{\mu}^{\mathrm{P}}(x)+\partial_{\mu}\left(I_{e} A_{\nu}^{\mathrm{P}}\right)(x) e^{\nu} \equiv\left(J_{e}\right)_{\mu}{ }^{\nu} A_{\nu}^{\mathrm{P}}
$$

with $I_{e}$ and $J_{e}$ defined in eq. (2.12), eq. (2.15). Its two-point function arises by integration over the Proca two-point function, that results in a multiplication of the Proca two-point kernel eq. (3.2) with the matrices $J_{e}(p)$ defined in eq. (2.16):

$$
M_{m}^{A_{\mu}(-e), A_{\mu^{\prime}}\left(e^{\prime}\right)}(p)=-E\left(e, e^{\prime}\right)(p)_{\mu \mu^{\prime}} \equiv-J_{e}(p)_{\mu}^{\nu} J_{e^{\prime}}(p)_{\mu^{\prime}} \nu^{\prime} \eta_{\nu \nu^{\prime}}
$$

Explicitly,

$$
E\left(e, e^{\prime}\right)(p)_{\mu \mu^{\prime}}=\left[\eta_{\mu \mu^{\prime}}-\frac{e_{\mu}^{\prime} p_{\mu^{\prime}}}{\left(p e^{\prime}\right)_{+}}-\frac{p_{\mu} e_{\mu^{\prime}}}{(p e)_{+}}+\frac{\left(e e^{\prime}\right) p_{\mu} p_{\mu^{\prime}}}{(p e)_{+}\left(p e^{\prime}\right)_{+}}\right]
$$

We have chosen the string $-e$ in the left argument in eq. (3.4) in order to avoid denominators $1 /(-p e)_{+}=-1 /(p e)_{-}$if the argument were $+e$. In particular, $E(e, e)(p)$ is the momentum space version of the operator $E_{e}$ in eq. (2.36).

It follows from eq. (3.4)

$$
M_{m}^{(\partial A)(-e),(\partial A)\left(e^{\prime}\right)}(p)=-p^{\mu} p^{\nu} E\left(e, e^{\prime}\right)(p)_{\mu \nu}=m^{2}\left(1-m^{2} \frac{\left(e e^{\prime}\right)}{(e p)_{+}\left(e^{\prime} p\right)_{+}}\right) .
$$

Therefore, the field $a(e, x):=-m^{-1} \partial_{\mu} A^{\mu}(e, x)$ is regular at $m=0$ and converges to the canonical scalar field $\varphi$.

For the string-localized Maxwell potential eq. (1.1), which has no covariant pointlocalized potential on the Hilbert space, one has to compute the two-point function by integration over the field strength whose two-point kernel is $M_{0}^{F_{\mu \nu}, F_{\kappa \lambda}}=-p_{\mu} p_{\kappa} \eta_{\nu \lambda}+p_{\nu} p_{\kappa} \eta_{\mu \lambda}+$ $p_{\mu} p_{\lambda} \eta_{\nu \kappa}-p_{\nu} p_{\lambda} \eta_{\mu \kappa}$. This gives the same formula eq. (3.4) except that the mass is zero and $p^{2}=0$.

This continuity property does not persist at $s>1$, see [16, 17], where the decoupling of the lower helicities is more subtle than at $s=1$. 


\subsection{Two-point functions of infinite-spin fields}

The two-point kernels of infinite spin fields eq. (2.3) given in terms of their intertwiners are

$$
M_{0}^{\phi_{u}(-e), \phi_{u}\left(e^{\prime}\right)}(p)=\int d \mu_{\kappa}(\vec{k}) \overline{u(-e, p)(\vec{k})} u\left(e^{\prime}, p\right)(\vec{k}) .
$$

For the standard field $\phi^{\kappa(0)}=\Phi^{\kappa(0)}$, we get

Proposition 3.1 (see also [12], chapter 4.1) The two-point kernel of $\phi^{\kappa(0)}(e, x)$ is

$$
M_{0}^{\phi^{\kappa(0)}(-e), \phi^{\kappa(0)}\left(e^{\prime}\right)}=\overline{\omega^{\kappa}(-e, p)} \omega^{\kappa}\left(e^{\prime}, p\right) \cdot J_{0}\left(\kappa R_{e, e^{\prime}, p}\right)
$$

where $q_{e}(p):=\frac{e}{(e p)_{+}}$and $R_{e, e^{\prime}, p}^{2}=-\left(q_{e}(p)-q_{e^{\prime}}(p)\right)^{2}$.

Notice that $q_{e}-q_{e^{\prime}}$ is orthogonal to $p$ because $\left(p q_{e}\right)=\left(p q_{e^{\prime}}\right)=1$, hence $q_{e}-q_{e^{\prime}}$ is spacelike and the argument of the Bessel function is real. The distributional nature of $R_{e, e^{\prime}, p}$ is absorbed by the Köhler factors, see Proposition 2.1.

Proof. Recall the definition of the Bessel functions

$$
\frac{1}{2 \pi} \int d \varphi e^{i \nu \varphi} e^{i z \cos \varphi}=i^{\nu} J_{\nu}(z)=i^{-\nu} J_{-\nu}(z)
$$

Thus, with $\widetilde{u}^{\kappa}$ given by eq. (2.8), the integral in eq. (3.7) can be performed:

$$
\int d \mu_{\kappa}(\vec{k}) e^{i \frac{\left(e E_{p}(\vec{k})\right)}{(e p)_{+}}} e^{-i \frac{\left(e^{\prime} E_{p}(\vec{k})\right)}{\left(e^{\prime} p\right)_{+}}}=\int d \mu_{\kappa}(\vec{k}) e^{-i \vec{k} \cdot \vec{f}}=J_{0}(\kappa|\vec{f}|)
$$

where $\vec{f}$ is the transverse (1-2-)part of the four-vector

$$
f=B_{p}^{-1}\left(\frac{e}{(e p)_{+}}-\frac{e^{\prime}}{\left(e^{\prime} p\right)_{+}}\right)=B_{p}^{-1}\left(q_{e}-q_{e^{\prime}}\right) .
$$

Because $\left(f p_{0}\right)=0$, one has $f^{2}=\left(f p_{0}\right)\left(f \xi_{0}\right)-|\vec{f}|^{2}=-|\vec{f}|^{2}$. The claim follows by multiplying with the Köhler factors.

Proposition 3.2 The two-point functions among $\Phi^{\kappa(r)}$ are given by their two-point kernels (no respective sums if $r=0$ or $r^{\prime}=0$ )

$$
\begin{aligned}
M_{0}^{\Phi^{\kappa(r)}(-e, v), \Phi^{\kappa\left(r^{\prime}\right)}\left(e^{\prime}, v^{\prime}\right)}= & \overline{\omega^{\kappa}(-e, p)} \omega^{\kappa}\left(e^{\prime}, p\right) \cdot \sum_{\nu= \pm r}\left(v \mathcal{E}_{ \pm}\right)^{r} \sum_{\nu^{\prime}= \pm r^{\prime}}\left(v \mathcal{E}_{ \pm}^{\prime}\right)^{r^{\prime}} . \\
& \cdot e^{-i\left(\nu+\nu^{\prime}\right) \alpha_{e, e^{\prime}, p} \cdot i^{\nu+\nu^{\prime}} J_{\nu+\nu^{\prime}}\left(\kappa R_{e, e^{\prime}, p}\right) .}
\end{aligned}
$$

Here, $R_{e, e^{\prime}, p}\left(\begin{array}{c}\cos \alpha_{e, e^{\prime}, p} \\ \sin \alpha_{e, e^{\prime}, p}\end{array}\right)$ parametrizes the 1-2-part of $B_{p}^{-1}\left(q_{e}(p)-q_{e^{\prime}}(p)\right)$.

Proof. By a direct computation, using eq. (2.32) and eq. (3.9).

Remark 3.3 The fields $\Phi^{\kappa(r)}$ mutually decouple in the limit $\kappa \rightarrow 0$, because $J_{\nu}(0)=\delta_{\nu, 0}$. Their limits as $\kappa \rightarrow 0$ coincide with the massless potentials $A^{(r, r)}$, i.e., the infinite-spin field "decays" into a direct sum of massless fields of every helicity ([12], chapter 4). 


\subsection{Massive fields of finite spin: definitions and properties}

Our aim is to approximate eq. (3.8) by higher-spin generalizations of eq. (3.4) and eq. (3.6) in the Pauli-Lubanski limit. We take stock of the relevant results in $[16,17]$, and supplement it by the crucial recursive formula Proposition 3.4.

We are going to work with the string-localized fields ${ }^{2} a_{\mu_{1} \ldots \mu_{r}}^{(s, r)}(e, x)$ defined in eq. (3.11) and eq. (3.12), and the string-localized fields $A^{(s, r)}(e, x)$ defined in eq. (3.24). All these fields are derived from the total field strengths $F_{\left[\mu_{1} \nu_{1}\right] \ldots\left[\mu_{s} \nu_{s}\right]}^{\mathrm{P}(s)}(x)$ of the point-localized massive "Proca" fields $A_{\mu_{1} \ldots \mu_{s}}^{\mathrm{P}(s)}(x)$ of spin $s[8]$. We start with the definition

$$
a_{\mu_{1} \ldots \mu_{s}}^{(s, s)}(e, x):=\left(\left(I_{e}\right)^{s} F_{\left[\mu_{1} \nu_{1}\right] \ldots\left[\mu_{s} \nu_{s}\right]}^{\mathrm{P}(s)}\right)(x) \cdot e^{\nu_{1}} \ldots e^{\nu_{s}}
$$

and the descending recursion

$$
-m \cdot a_{\mu_{1} \ldots \mu_{r}}^{(s, r)}(e, x):=\partial^{\mu} a_{\mu \mu_{1} \ldots \mu_{r}}^{(s, r+1)}(e, x) .
$$

$a^{(s, s)}$ differs from $A^{\mathrm{P}(s)}$ by derivatives of $a^{(s, r)}(r<s)$ ([16], Proposition 3.5), so that it is also a potential for the field strength $F^{\mathrm{P}(s)}$. Unlike the Proca potential, the field strength and hence also the string-localized potential $a^{(s, s)}$ and its escort fields $a^{(s, r)}$ are regular in the limit $m \rightarrow 0$ at fixed spin $s$. The discrepancy between these limits and potentials $A^{(r)}$ for the massless field strengths $F^{(r)}$ was studied in [16].

Let us identify some general patterns. The composition of the operations

$$
(\text { contraction with } e) \circ(\text { string integration }) \circ(\text { curl }),
$$

applied to each index of $A^{\mathrm{P}(s)}$ in the definition eq. (3.11), is the matrix operator $\left(J_{e}\right)_{\mu}{ }^{\nu}=$ $\delta_{\mu}^{\nu}+I_{e} e^{\nu} \partial_{\mu}$ already seen in eq. (2.15) and eq. (3.3). Thus, eq. (3.11) and eq. (3.12) become

$$
\begin{aligned}
& a_{\mu_{1} \ldots \mu_{s}}^{(s, s)}(e, x)=\left(J_{e}\right)_{\mu_{1}}^{\nu_{1}} \ldots\left(J_{e}\right)_{\mu_{s}}^{\nu_{s}} A_{\nu_{1} \ldots \nu_{s}}^{\mathrm{P}(s)}(x), \\
& a_{\mu_{1} \ldots \mu_{r}}^{(s, r)}(e, x)=\left(J_{e}\right)_{\mu_{1}}^{\nu_{1}} \ldots\left(J_{e}\right)_{\mu_{r}}^{\nu_{r}}\left(m e^{\nu_{r+1}} I_{e}\right) \ldots\left(m e^{\nu_{s}} I_{e}\right) A_{\nu_{1} \ldots \nu_{s}}^{\mathrm{P}(s)}(x),
\end{aligned}
$$

Because $J_{\lambda e}=J_{e}(\lambda>0)$, all $a^{(s, r)}(e, x)$ are homogeneous distributions in $e$.

The following formula for the $e$-dependence of $a^{(s, s)}(e, x)$ was displayed in ([16], Corollary 3.3) with an erroneous factor $m^{-1}$ :

$$
\partial_{e^{\mu}} a_{\mu_{1} \ldots \mu_{s}}^{(s, s)}(e, x)=\sum_{i=1}^{s} \partial_{\mu_{i}} I_{e} a_{\ldots \mu_{i-1} \mu \mu_{i+1} \ldots}^{(s, s)}(e, x) .
$$

It follows from the definition of $a^{(s, s)}$ and the identities eq. (2.18) and eq. (2.13).

Proposition 3.4 The descending defining recursion eq. (3.12) is inverted by the ascending recursion, involving the variation of the direction e,

$$
-m(s-r) \cdot a_{\mu \mu_{1} \ldots \mu_{r}}^{(s, r+1)}(e, x)=\sum_{i=1}^{r} \partial_{\mu_{i}} a_{\ldots \mu_{i-1} \mu \mu_{i+1} \ldots}^{(s, r)}+\left(e \partial_{x}\right) \partial_{e^{\mu}} a_{\mu_{1} \ldots \mu_{r}}^{(s, r)}(e, x) .
$$

In particular, the r.h.s. is completely symmetric in $\mu, \mu_{1}, \ldots, \mu_{r}$.

This result conversely exhibits also the $e$-dependence of $a_{\mu_{1} \ldots \mu_{r}}^{(s, r)}(e, x)$ via eq. (3.16).

\footnotetext{
${ }^{2}$ The first superscript $s$ was suppressed in $[16,17]$, where we worked at fixed $s$.
} 
Proof. ${ }^{3}$ Apply $\partial^{\mu_{s}} \ldots \partial^{\mu_{r+1}}$ to eq. (3.14) and use the defining recursion eq. (3.12). This gives

$$
\partial_{e^{\mu}} a_{\mu_{1} \ldots \mu_{r}}^{(s, r)}(e, x)=m(s-r) \cdot I_{e} a_{\mu \mu_{1} \ldots \mu_{r}}^{(s, r+1)}(e, x)+\sum_{i=1}^{r} \partial_{\mu_{i}} I_{e} a_{\ldots \mu_{i-1} \mu \mu_{i+1} \ldots}^{(s, r)}
$$

which can be solved for $a_{\mu \mu_{1} \ldots \mu_{r}}^{(s, r+1)}(e, x)$ using eq. (2.13).

We also report the identities

Proposition 3.5 (see [16], Proposition 3.6) For $r \geq 2$, resp. $r \geq 0$

$$
\eta^{\kappa \lambda} a_{\kappa \lambda \mu_{3} \ldots \mu_{r}}^{(s, r)}=-a_{\mu_{3} \ldots \mu_{r}}^{(s, r-2)}, \quad e^{\kappa} a_{\kappa \mu_{2} \ldots \mu_{r}}^{(s, r)}=0 .
$$

Proof. By inspection of eq. (3.13), using that $A^{\mathrm{P}(s)}$ is traceless and conserved.

We now turn to two-point functions.

The intertwiner for the $(m, s)$ Proca potential is given by the $s$-fold tensor product of the standard intertwiner for spin 1 [28-30], preceded by the projection onto the traceless symmetric subrepresentation $(=\operatorname{spin} s$ representation) of the tensor product of spin 1 representations of the little group $\mathrm{SO}(3)$ :

$$
u_{n}^{\mathrm{P}(s)}(p)=\left(B_{p} E_{3}\right)^{\otimes s} T_{n},
$$

where $T_{n}, n=1, \ldots, 2 s+1$, is an orthonormal basis of traceless symmetric tensors in $\left(\mathbb{R}^{3}\right)^{\otimes s}$, and $E_{3}: \mathbb{R}^{3} \rightarrow \mathbb{R}^{4}$ the standard embedding into Minkowski space.

The resulting two-point kernel of the Proca field is (in the notations introduced in section 2.2 and section 3.1 )

Proposition 3.6 (see [11] and [16], section 2.1)

$$
M_{m}^{A^{\mathrm{P}(s)}(v), A^{\mathrm{P}(s)}\left(v^{\prime}\right)}=(-1)^{s} \sum_{2 n \leq s} \beta_{n}^{s} \cdot\left[(v \pi v)\left(v^{\prime} \pi v^{\prime}\right)\right]^{n} \cdot\left(v \pi v^{\prime}\right)^{s-2 n} .
$$

Here, $\pi_{\mu \nu}=\eta_{\mu \nu}-\frac{p_{\mu} p_{\nu}}{m^{2}}$, and $(v \pi v)$ etc. are the contractions with $v$ resp. $v^{\prime}$. The coefficients $\beta_{n}^{s}=\frac{1}{4^{n} n !} \frac{s !}{(s-2 n) !} \frac{1}{\left(\frac{1}{2}-s\right)_{n}}$ (ensuring the tracelessness) are the coefficients of the hypergeometric function

$$
F_{s}(z) \equiv \sum_{2 n \leq s} \beta_{n}^{s} z^{n}={ }_{2} F_{1}\left(\frac{-s}{2}, \frac{1-s}{2} ; \frac{1}{2}-s ; z\right) .
$$

This function is in fact a polynomial of order $\left\lfloor\frac{s}{2}\right\rfloor$, because either $\frac{-s}{2}$ or $\frac{1-s}{2}$ is a non-positive integer.

The two-point kernel of the massive spin $s$ string-localized potential $a^{(s, s)}$ follows from the definition eq. (3.11):

Proposition 3.7 (see [16], section 3)

$$
M_{m}^{a^{(s, s)}(-e, v), a^{(s, s)}\left(e^{\prime}, v^{\prime}\right)}=(-1)^{s} \sum_{2 n \leq s} \beta_{n}^{s} \cdot\left[(v E v)\left(v^{\prime} E^{\prime \prime} v^{\prime}\right)\right]^{n} \cdot\left(v E^{\prime} v^{\prime}\right)^{s-2 n} .
$$

Here, $E \equiv E(e, e)(p), E^{\prime} \equiv E\left(e, e^{\prime}\right)(p), E^{\prime \prime} \equiv E\left(e^{\prime}, e^{\prime}\right)(p)$ as in eq. (3.4).

\footnotetext{
${ }^{3} \mathrm{C}$. Gaß has suggested this simpler proof of a stronger statement than in a previous version.
} 
From eq. (3.21), one gets the correlations of all escort fields $a^{(s, r)}$ by descending in $r$ with the defining recursion eq. (3.12). It turns out to be more convenient to descend directly to $r=0: a^{(s, 0)}(e, x)=\frac{1}{s !}(-m)^{-s}\left(\partial_{x} \partial_{v}\right) a^{(s, s)}(e, x, v)$, and then use the ascending recursion eq. (3.15). This strategy will allow to study limits of $a_{\mu_{1} \ldots \mu_{r}}^{(s, r)}$ for fixed $r$ while $s$ increases.

Taking the divergence in all indices via $\left(\partial_{x} \partial_{v}\right)=-i\left(p \partial_{v}\right)$ and $\left(\partial_{x^{\prime}} \partial_{v^{\prime}}\right)=+i\left(p \partial_{v^{\prime}}\right)$, just amounts to putting $v=v^{\prime}=p / m$ in the two-point kernel eq. (3.21). One gets the two-point kernel of the string-localized "scalar" escort field $a^{(s, 0)}$ :

Proposition 3.8 (see [16], section 3)

$$
M_{m}^{a^{(s, 0)(-e)}, a^{(s, 0)}\left(e^{\prime}\right)}=(-1)^{s} m^{-2 s} \sum \beta_{n}^{s} \cdot\left[(p E p)\left(p E^{\prime \prime} p\right)\right]^{n} \cdot\left(p E^{\prime} p\right)^{s-2 n},
$$

where $(p E p)=E(e, e)(p)=-m^{2}\left(1-m^{2} q_{e}^{2}\right)$, and similar for $\left(p E^{\prime} p\right),\left(p E^{\prime \prime} p\right)$.

We rewrite eq. (3.22) as

$$
M_{m}^{a^{(s, 0)}(-e), a^{(s, 0)}\left(e^{\prime}\right)}=\left(1-m^{2}\left(q_{e} q_{e^{\prime}}\right)\right)^{s} \cdot F_{s}\left(z_{m}\left(q_{e}, q_{e^{\prime}}\right)\right) \equiv P_{m}^{s}\left(q_{e}, q_{e^{\prime}}\right)
$$

where $z_{m}\left(q, q^{\prime}\right)=\frac{\left(1-m^{2} q^{2}\right)\left(1-m^{2} q^{\prime 2}\right)}{\left(1-m^{2}\left(q q^{\prime}\right)\right)^{2}}$. Notice that $P_{m}^{s}\left(q_{e}, q_{e^{\prime}}\right)$ is a polynomial in $q_{e}, q_{e^{\prime}}$, hence immediately well-defined as a distribution.

From this, one may obtain the two-point kernels for $a^{(s, r)}$ by ascending with eq. (3.15), using eq. (2.16) and eq. (2.18) in momentum space.

We have also defined string-localized fields $A^{(s, r)}$ that decouple in the massless limit at fixed $s$, and become potentials for the massless field strengths $F^{(r)}$ of helicity $\pm r$ :

Definition 3.9 (see [16], Proposition 3.8)

$$
A^{(s, r)}(e, x, v):=N_{0}^{(s, r)} \cdot \sum_{2 k \leq r} \gamma_{k}^{r} \cdot\left(-E_{e}(v)\right)^{k} a^{(s, r-2 k)}(e, x, v)
$$

with $E_{e}$ as in eq. (2.36). The coefficients are $\gamma_{k}^{r}=\frac{1}{4^{k} k !} \frac{r !}{(r-2 k) !} \frac{1}{(1-r)_{k}}$, and $N_{0}^{(s, r)}:=$ $\left[\left(\begin{array}{l}s \\ r\end{array}\right) \frac{\Gamma\left(\frac{1}{2}+s\right) \Gamma(1+r)}{\Gamma\left(\frac{1}{2}+\frac{r+s}{2}\right) \Gamma\left(1+\frac{r+s}{2}\right)}\right]^{\frac{1}{2}}$.

Proposition 3.10 (see [16], Corollary 3.10) In the limit $m \rightarrow 0$ at fixed $s$ and $r$, the string-localized fields $A^{(s, r)}(e, x)$ mutually decouple, and are potentials for the point-localized massless field strengths $F^{(r)}(x)$ associated with the Wigner representations of helicities $\pm r$. In particular, at $m=0$ they no longer depend on $s$, they are traceless, conserved, and satisfy $e^{\mu} A_{\mu \mu_{2} \ldots \mu_{r}}^{(s, r)}=0$.

\section{The Pauli-Lubanski limit}

After these preparations, we turn to the Pauli-Lubanski limit. The limit of the "scalar" escort fields $a^{(s, 0)}(e, x)$ is rather easy. It relies on two lemmas. 
Lemma 4.1 For $s \in \mathbb{N}$, the identity of polynomials of degree $\left\lfloor\frac{s}{2}\right\rfloor$

$$
{ }_{2} F_{1}\left(\frac{-s}{2}, \frac{1-s}{2} ; \frac{1}{2}-s ; z\right)=\frac{\Gamma\left(1+\frac{s}{2}\right) \Gamma\left(\frac{1}{2}+\frac{s}{2}\right)}{\Gamma\left(\frac{1}{2}+s\right)} \cdot{ }_{2} F_{1}\left(\frac{-s}{2}, \frac{1-s}{2} ; 1 ; 1-z\right)
$$

holds. We henceforth abbreviate this identity as

$$
F_{s}(z)=F_{s}(1) \cdot G_{s}(1-z)
$$

in accord with the previous notation eq. (3.20). In particular, $F_{s}(1)=\frac{\Gamma\left(1+\frac{s}{2}\right) \Gamma\left(\frac{1}{2}+\frac{s}{2}\right)}{\Gamma\left(\frac{1}{2}+s\right)}$ $\equiv\left(N_{0}^{(s, 0)}\right)^{-2}$. For large $s$, this decays asymptotically like $F_{s}(1) \approx 2^{-s} \sqrt{\pi s}$.

Proof. The identity is the formula 2.9(43) in [7] with parameters $a=\frac{-s}{2}, b=\frac{1-s}{2}$, $c=\frac{1}{2}-s$. The value $F_{s}(1)$ follows because $G_{s}(0)=1$. The asymptotic form follows by Stirling's approximation of the $\Gamma$ function.

Lemma 4.2 In the limit $s \rightarrow \infty$, the pointwise limit holds

$$
\lim _{s \rightarrow \infty} G_{s}\left(-\frac{u^{2}}{s^{2}}\right) \equiv \lim _{s \rightarrow \infty} F_{1}\left(\frac{-s}{2}, \frac{1-s}{2} ; 1 ;-\frac{u^{2}}{s^{2}}\right)=J_{0}(u) .
$$

Proof. The power series expansion reads

$$
G_{s}\left(-\frac{u^{2}}{s^{2}}\right)=\sum_{k \geq 0} \frac{(-s)_{2 k}}{4^{k} k !^{2}}\left(-\frac{u^{2}}{s^{2}}\right)^{k} \rightarrow \sum_{k \geq 0} \frac{1}{k !^{2}}\left(-\frac{u^{2}}{4}\right)^{k}=J_{0}(u),
$$

where the limit of the coefficients is taken separately for each $k$. The pointwise convergence in $u$ follows by absolute convergence of the sums.

Now we turn to the fields.

Proposition 4.3 In the Pauli-Lubanski limit, the two-point kernel of the rescaled scalar escort fields $N_{0}^{(s, 0)} \cdot a^{(s, 0)}(e)$ converges to $J_{0}\left(\kappa \sqrt{-\left(q_{e}(p)-q_{e^{\prime}}(p)\right)^{2}}\right)$. Since $J_{0}(z)$ is a power series in $z^{2}$, the two-point kernel is a power series in $q_{e}$ and $q_{e^{\prime}}$. The convergence is pointwise, i.e., it holds formally for fixed values of $q_{e}$ and $q_{e^{\prime}}$, and more precisely for fixed test functions in $e, e^{\prime}$ and $p$, on whose support $\left(q_{e}(p)-q_{e^{\prime}}(p)\right)^{2}$ is bounded.

Proof. Using Lemma 4.1, we rewrite eq. (3.23) as

$$
M_{m}^{a^{(s, 0)}(-e), a^{(s, 0)}\left(e^{\prime}\right)}=\left(1-m^{2}\left(q_{e} q_{e^{\prime}}\right)\right)^{s} \cdot F_{s}(1) G_{s}\left(1-z_{m}\left(q_{e}, q_{e^{\prime}}\right)\right) .
$$

The prefactor $\left(1-\frac{\kappa^{2}\left(q q^{\prime}\right)}{s(s+1)}\right)^{s}$ converges separately to 1 by Euler's formula. The claim then follows by Lemma 4.2 .

Comparing the limit obtained in Proposition 4.3 with the two-point kernel eq. (3.8) of the standard string-localized field $\phi^{\kappa(0)}$, one notes that the Köhler factors are missing. But they can be produced by applying the operators $\left(1-m \sqrt{-e^{2}} I_{e}\right)^{s}$ before the limit is taken. Namely, by Euler's formula, $\omega^{\kappa}(e, p)=e^{-i \kappa \frac{\sqrt{-e^{2}}}{(e p)_{+}}}$is the limit of $\left(1-i m \frac{\sqrt{-e^{2}}}{(e p)_{+}}\right)^{s}$, and $\frac{i}{(e p)_{+}}$ is the momentum space version of the string-integration $I_{e}$. 
Corollary 4.4 The standard string-localized field $\phi^{\kappa(0)}(e, x)$ is, up to unitary equivalence, the Pauli-Lubanski limit of $N_{0}^{(s, 0)} \cdot\left(1-m \sqrt{-e^{2}} I_{e}\right)^{s} a^{(s, 0)}(e, x)$.

Remark 4.5 The convergence of the two-point kernels is much easier to see than that of the intertwiners, because the former is basis independent. The reason is that "convergence" of vectors on different Hilbert spaces makes only sense with a suitable inductive limit (a sequence of embeddings of the Hilbert spaces). For the case at hand, this inductive limit of the representation spaces of the massive little group $\mathrm{SO}(3)$ is described in appendix A, in such a way that the matrix elements converge to a representation of the massless little group E(2), and this extends to the induced Wigner representation [14].

With the given inductive identification of bases, one should be able to prove the convergence of intertwiners up to unitary equivalence. We refrain from doing this because the two-point function uniquely specifies a free field, and hence we may conclude the convergence of the intertwiners and of the fields up to unitary equivalence. In this sense, we may say

$$
\lim _{\kappa} N_{0}^{(s, 0)} \cdot\left(1-m \sqrt{-e^{2}} I_{e}\right)^{s} a^{(s, 0)}(e, x)=\phi^{\kappa(0)}(e, x) .
$$

Proposition 4.6 The fields $N_{0}^{(s, 0)} \cdot\left(1-m \sqrt{-e^{2}} I_{e}\right)^{s} a^{(s, r)}(e, x)$ converge in the PauliLubanski limit (in the same sense as specified in Remark 4.5) to the infinite-spin fields $\phi^{\kappa(r)}(e, x)$ defined in Definition 2.4.

Proof. (i) The recursion eq. (3.15) for $a^{(s, r)}$ implies the recursion

$$
-\kappa \widetilde{\phi}^{\kappa(r+1)}(e, x, v)=\left(r \cdot\left(v \partial_{x}\right)+\left(e \partial_{x}\right)\left(v \partial_{e}\right)\right) \widetilde{\phi}^{\kappa(r)}(e, x, v)
$$

for the limits of $N_{s}^{(s, 0)} \cdot a^{(s, r)}$, because for each fixed $r, m(s-r)$ can be replaced by $\kappa$. Restoring the Köhler factors of the intertwiners by means of the operators $\left(1-m \sqrt{-e^{2}} I_{e}\right)^{s}$ as in Proposition 4.3, implies the recursion eq. (2.27) for the limits of $N_{0}^{(s, 0)} \cdot\left(1-m \sqrt{-e^{2}} I_{e}\right)^{s} a^{(s, r)}(e, x)$. The claim follows, because eq. (2.27) defines $\phi^{\kappa(r)}$.

We now study the Pauli-Lubanski limit of the fields $A^{(s, r)}$, defined in Definition 3.9. Using the trace identity in eq. (3.17), we rewrite eq. (3.24) as

$$
A^{(s, r)}(e, x)=\frac{N_{0}^{(s, r)}}{N_{0}^{(s, 0)}} \cdot\left(S_{r} \sum_{2 k \leq r} \gamma_{k}^{r} \cdot\left(\left(J_{e} \otimes J_{e}\right) \eta \circ \eta^{t}\right)^{\otimes k}\right) \cdot N_{0}^{(s, 0)} a^{(s, r)}(e, x),
$$

regarding $a^{(s, r)}$ as a tensor in $\left(\mathbb{R}^{4}\right)^{\otimes r}$ of which pairs of indices are contracted with $\eta$ and restored by $E_{e}=\left(J_{e} \otimes J_{e}\right) \eta$, and the result in $\left(\mathbb{R}^{4}\right)^{\otimes r}$ is symmetrized.

Corollary 4.7 In the Pauli-Lubanski limit, the fields $\left(1-m \sqrt{-e^{2}} I_{e}\right)^{s} A^{(s, r)}(e, x)$ converge (in the same sense as specified in Remark 4.5) to the infinite-spin fields $\Phi^{\kappa(r)}(e, x)$ defined in Definition 2.7.

Proof. One easily sees from the formula given in Definition 3.9 that the prefactor $\frac{N_{0}^{(s, r)}}{N_{0}^{(s, 0)}}$ converges to $2^{r / 2}$ as $s \rightarrow \infty$. The operator does not depend on $s$ and $m$ and commutes with $\left(1-m \sqrt{-e^{2}} I_{e}\right)^{s}$, and $N_{0}^{(s, 0)} \cdot\left(1-m \sqrt{-e^{2}} I_{e}\right)^{s} a^{(s, r)}(e, x)$ converges to $\phi^{\kappa(r)}$ by Proposition 4.6. Thus, the limit of eq. (4.5) is equivalent to eq. (2.33). 


\section{$5 \quad$ Stress-energy tensors}

In [16], we have introduced several stress-energy tensors for the $(m, s)$ fields that all yield the correct infinitesimal generators

$$
P_{\sigma}=\int_{x_{0}=t} d^{3} \vec{x} T_{0 \sigma}, \quad M_{\sigma \tau}=\int_{x_{0}=t} d^{3} \vec{x}\left(x_{\sigma} T_{0 \tau}-x_{\tau} T_{0 \sigma}\right)
$$

of the Poincaré group. They differ by derivative terms that vanish upon the integrations eq. (5.1). We display here: the point-localized "reduced" stress-energy tensor

$$
T_{\rho \sigma}^{(s) \operatorname{red}}(x)=-\frac{1}{4}(-1)^{s}: A^{\mathrm{P}(s) \mu_{1} \ldots \mu_{s}} \stackrel{\leftrightarrow}{\partial_{\rho}} \stackrel{\leftrightarrow}{\partial_{\sigma}} A_{\mu_{1} \ldots \mu_{s}}^{\mathrm{P}(s)}:(x)+\partial^{\mu} \Delta T_{\rho \sigma ; \mu}^{(s) \mathrm{red}}
$$

and the string-localized "regular" stress-energy tensor

$$
\begin{aligned}
t_{\rho \sigma}^{(s) \mathrm{reg}}\left(e_{1}, e_{2}, x\right) & =\sum_{r \leq s}\left(\begin{array}{c}
s \\
r
\end{array}\right) t_{\rho \sigma}^{(s, r)}\left(e_{1}, e_{2}, x\right) \\
t_{\rho \sigma}^{(s, r)}\left(e_{1}, e_{2}, x\right) & =-\frac{1}{4}(-1)^{r}: a^{(s, r) \mu_{1} \ldots \mu_{r}}\left(e_{1}\right) \overleftrightarrow{\partial_{\rho}} \overleftrightarrow{\partial_{\sigma}} a_{\mu_{1} \ldots \mu_{r}}^{(s, r)}\left(e_{2}\right):(x)+\partial^{\mu} \Delta t_{\rho \sigma ; \mu}^{(r) \mathrm{reg}} .
\end{aligned}
$$

The derivative terms $\partial^{\mu} \Delta T_{\rho \sigma ; \mu}$ do not affect the momentum generators, but they have to be added to get the correct infinitesimal Lorentz transformations. Explicit expressions can be found in [16]. We do not need them at this point, see however Example 5.5. The tensors $t^{(s, r)}$ are separately conserved, but only their sum eq. (5.3) generates the correct Poincaré transformations.

We have also given massless stress-energy tensors

$$
\begin{aligned}
T_{\rho \sigma}^{(s), m=0}\left(e_{1}, e_{2}, x\right) & =\sum_{r \leq s}\left(\begin{array}{c}
s \\
r
\end{array}\right) T_{\rho \sigma}^{(s, r), m=0}\left(e_{1}, e_{2}, x\right), \\
T_{\rho \sigma}^{(s, r), m=0}\left(e_{1}, e_{2}, x\right) & =-\frac{1}{4}(-1)^{r}: A^{(s, r) \mu_{1} \ldots \mu_{r}}\left(e_{1}\right) \stackrel{\leftrightarrow}{\partial_{\rho}} \stackrel{\leftrightarrow}{\partial_{\sigma}} A_{\mu_{1} \ldots \mu_{r}}^{(s, r)}\left(e_{2}\right):(x)+\partial^{\mu} \Delta T_{\rho \sigma ; \mu}^{(s, r), m=0},
\end{aligned}
$$

that, in spite of the definition of the string-localized fields $A^{(s, r)}$ as massless limits of fields on the massive Fock space of $\operatorname{spin} s$ (in terms of $a^{\left(s, r^{\prime} \leq r\right)}$, see Definition 3.9), do not depend on $s$. At $m=0$, eq. (5.4) describes the decoupling of the massive spin $s$ representation into a direct sum of helicity representations $[16,17]$.

Let us discuss the possible role of these tensors in the Pauli-Lubanski limit. The pointlocalized reduced stress-energy tensor $T^{(s) \text { red }}$ does not admit a massless limit because of inverse powers $m^{-4 s}$ in the two-point function. The string-localized regular stress-energy tensor $t^{(s) \text { reg }}$ becomes in the Pauli-Lubanski limit an infinite sum over $r \leq s \rightarrow \infty$ of terms $t^{(s, r)}$, that each converge to zero (due to the explicit factor $F_{s}(1) \approx 2^{-s}$ present in every two-point function $\left(\Omega, a^{(s, r)} a^{\left(s, r^{\prime}\right)} \Omega\right)$ ). Yet, the sum is not zero and is still a valid stressenergy tensor, but it cannot be expressed as a sum of limits of $t^{(s, r)}$. (These and other interesting features are nicely illustrated by the expectation values of the energy density and the pressure in thermal states at inverse temperature $\beta$, cf. section 5.3.4. E.g., while the contribution of each $r$ to the thermal energy goes to zero, the sum over $r$ diverges like $2 s+1$.) 
Eq. (5.4) seems to be better suited for the Pauli-Lubanski limit, because each term $T^{(s, r)}$ has a limit. At fixed $s$, the massless stress-energy tensor $T^{(s), m=0}$ is the limit of massive conserved tensors $T^{(s), m}[16]$. The latter differ from $t^{(s) \mathrm{reg}}$, apart from irrelevant terms that do not affect the Poincaré generators, by further terms that do disturb the generators, and that decay like $O(m)$ at fixed $s$ ([16], Propositions 4.5 and 4.6). But such terms may grow with $s$, so that it is difficult to keep control, whether the Pauli-Lubanski limit produces the correct generators.

Our main result in this section computes the stress-energy tensor directly in the infinitespin representation:

Proposition 5.1 Let $\Phi^{\kappa(r)}(e, x)$ be the string-localized fields defined in Definition 2.7 (that may be obtained as Pauli-Lubanski limits by Corollary 4.7). The conserved symmetric tensor

$$
\begin{aligned}
T_{\rho \sigma}^{\kappa}(e, x) & =\sum_{r=0}^{\infty} T_{\rho \sigma}^{\kappa(r)}(e, x), \\
T_{\rho \sigma}^{\kappa(r)}(e, x) & =-\frac{1}{4}(-1)^{r}: \Phi^{\kappa(r) \mu_{1} \ldots \mu_{r}}(e) \stackrel{\leftrightarrow}{\partial_{\rho}} \stackrel{\leftrightarrow}{\partial_{\sigma}} \Phi_{\mu_{1} \ldots \mu_{r}}^{\kappa(r)}(e):(x)+\partial^{\mu} \Delta T_{\rho \sigma ; \mu}^{\kappa(r)}
\end{aligned}
$$

is a string-localized stress-energy tensor for the infinite-spin representation. An expression for $\Delta T_{\rho \sigma ; \mu}^{\kappa(r)}$ will be given in eq. (5.13).

The proof in section 5.2 exhibits $\Delta T^{\kappa(r)}$ as a sum of two pieces. The "second piece" $\Delta_{2} T^{\kappa(r)}$ is absent at finite $s$ and must be identified with the accumulation of the above-mentioned uncontrolled errors.

Remark 5.2 In order to get the correct generators, the two string-localized fields in the Wick product have to be taken with $e_{1}=-e_{2}$, see Remark 5.7. Mund has recently shown that the Wick product with parallel strings is well-defined as a distribution in $x$ and $e$.

The problematic issue with eq. (5.5) is instead the infinite sum over $r$. Because the fields $\Phi^{\kappa(r)}$ do not decouple (see eq. (2.36)), correlation functions and matrix elements involving $T^{\kappa}$ may be divergent sums. Recall from Remark 2.10 that each $T^{\kappa(r)}$ will have a non-vanishing expectation value in a state with sharp magnetic quantum number $n \in \mathbb{Z}$. The consequences of this feature for two-point functions and commutators of $T^{\kappa}$ with the fields $\Phi^{\kappa(r)}$ will be sketched in section 5.3.

\subsection{Quantum stress-energy tensors}

We obtained Proposition 5.1 with a new systematic strategy to find stress-energy tensors for free quantum fields, that does not refer to a classical action principle. Instead, it is intrinsically based on the Wigner representation theory, along with a choice of intertwiners that allow to "decompose" the (global) generators into integrals over localized densities.

We first outline the general strategy, that is flexible enough to include also point- and string-localized finite-spin tensor fields and Dirac fields. The stress-energy tensors eq. (5.2), eq. (5.3) and eq. (5.4) could have been found by this strategy.

Applied to the infinite-spin case (section 5.2), it does not use the Pauli-Lubanski approximation, i.e., it proceeds directly in the limit, using just the results of Proposition 2.9. 
To keep the argument transparent, we present only the case of bosonic hermitean fields, where the $u$ - and $v$-intertwiners multiplying creation and annihilation operators are complex conjugates of each other.

We start with the familiar global "second quantization" formula for the momentum operator

$$
P_{\sigma}=\int d \mu_{m}(p) \sum_{n} p_{\sigma} a_{n}^{*}(p) a_{n}(p)
$$

where the sum extends over an orthonormal basis of the representation space $\mathcal{H}_{d}$ of the unitary representation $d$ of the little group. We write this as

$$
P_{\sigma}=\int d \mu_{m}\left(p_{1}\right) d \mu_{m}\left(p_{2}\right) \sum_{n_{1} n_{2}} p_{1 \sigma} a_{n_{1}}^{*}\left(p_{1}\right) \delta_{n_{1} n_{2}}(2 \pi)^{3} \delta\left(\vec{p}_{1}-\vec{p}_{2}\right)\left(p_{10}+p_{20}\right) a_{n_{2}}\left(p_{2}\right),
$$

and insert $(2 \pi)^{3} \delta\left(\vec{p}_{1}-\vec{p}_{2}\right)=\int d^{3} \vec{x} e^{-i\left(\vec{p}_{1}-\vec{p}_{2}\right) \vec{x}}=\int d^{3} \vec{x} e^{i\left(p_{1}-p_{2}\right) x}$, which is independent of $x^{0}$. Separating the factors that depend on $p_{1}$ and on $p_{2}$, respectively, and interchanging the $x$ - and $p$-integrations, one gets an $\vec{x}$-integral over the product of (derivatives of) two expressions $\int d \mu_{m}\left(p_{1}\right) e^{i p_{1} x} a_{n_{1}}^{*}\left(p_{1}\right)$ and $\int d \mu_{m}\left(p_{2}\right) e^{-i p_{2} x} a_{n_{2}}\left(p_{2}\right)$. These are of course not the creation and annihilation parts of a local and covariant quantum field, by the wellknown problem of the nonlocal Wigner "rotations", that is the reason why one has to use intertwiners in Wigner quantization [28-30].

So, let there be a (possibly reducible) representation $D$ of the Lorentz group and intertwiners $u_{M n}(p)$ satisfying

$$
u(\Lambda p)=\left(D(\Lambda) \otimes d\left(W_{\Lambda, p}\right)\right) u(p) \quad\left(\Lambda \in \mathrm{SO}(1,3)_{+}^{\uparrow}\right)
$$

(generalizing eq. (2.4) and eq. (2.25); $D(\Lambda)$ may include the action on a string variable), and that fulfill the completeness relation

$$
g^{M N} u_{M n_{1}}(p) \overline{u_{N n_{2}}(p)}=\delta_{n_{1} n_{2}}
$$

with a suitable "metric" $g^{M N}$. In order for eq. (5.8) to be fulfilled, $D(\Lambda)$ may have to be a direct sum of tensor representations (in the bosonic case), and hence $g$ a corresponding direct sum of tensor products of $\eta$; we present examples for such $u_{M n}$ below (Example 5.5 and section 5.2).

Inserting the partition of unity eq. (5.8) into the previous expression, we get

$$
P_{\sigma}=-\frac{1}{2} g^{M N} \int d^{3} \vec{x} \phi_{M}^{+}(x) \overleftrightarrow{\partial_{0}} \stackrel{\leftrightarrow}{\partial_{\sigma}} \phi_{N}^{-}(x)=-\frac{1}{4} g^{M N} \int d^{3} \vec{x}: \phi_{M} \stackrel{\leftrightarrow}{\partial_{0}} \overleftrightarrow{\partial_{\sigma}} \phi_{N}:(x),
$$

where $\phi_{M}^{+}(x)=\int d \mu_{m}(p) e^{i p x} u_{M n}(p) a_{n}^{*}(p)$, and $\phi_{M}^{-}(x)$ its hermitean conjugate. $\phi_{M}(x)=$ $\phi_{M}^{+}(x)+\phi_{M}^{-}(x)$ is a covariant field, point-localized or string-localized according to the choice of the intertwiners. The second equality holds by symmetry of the Wick product and because the operator $\overleftrightarrow{\partial_{0}}$ vanishes on the creation-creation and annihilation-annihilation parts of the Wick product thanks to $p_{10}=p_{20}$. 
The last expression is the desired local representation of eq. (5.6). The integrand

$$
\widetilde{T}_{\rho \sigma}(x)=-\frac{1}{4} g^{M N}: \phi_{M} \overleftrightarrow{\partial_{\rho}} \overleftrightarrow{\partial_{\sigma}} \phi_{N}:(x)
$$

is a first candidate for a stress-energy tensor, that by construction produces the correct generators $P_{\sigma}$ of translations. Of course, this construction is only unique up to terms that vanish upon the $\vec{x}$-integration, and we shall see presently that we need to add such terms in order to produce also the correct Lorentz generators.

We obtain the global form of the Lorentz generators from the transformation law of the creation operators:

$$
U(\Lambda) a_{n}^{*}(p) U(\Lambda)^{*}=a_{m}^{*}(\Lambda p) d_{m n}\left(W_{\Lambda, p}\right) \quad\left(\Lambda \in \mathrm{SO}(1,3)_{+}^{\uparrow}\right) .
$$

Infinitesimally:

$$
i\left[M_{\sigma \tau}, a_{n}^{*}(p)\right]=\left(\delta_{n n^{\prime}}\left(p_{\sigma} \partial_{p^{\tau}}-p_{\tau} \partial_{p^{\sigma}}\right)+d\left(\omega_{\sigma \tau}\right)_{n n^{\prime}}^{t}\right) a_{n^{\prime}}^{*}(p),
$$

where $\omega_{\sigma \tau}$ is the infinitesimal Wigner "rotation". The latter depends on the choice of the standard "boosts"; we do not display it because it is going to cancel anyway. What matters is that $d\left(\omega_{\sigma \tau}\right)$ is anti-hermitean on $\mathcal{H}_{d}$ because the representation $d$ is unitary, hence $-i\left(\mathbb{I}_{d} p \wedge \partial_{p}+d(\omega)^{t}\right)_{\sigma \tau} \equiv-i\left(\mathbb{I}_{d}\left(p_{\sigma} \partial_{p^{\tau}}-p_{\tau} \partial_{p^{\sigma}}\right)+d\left(\omega_{\sigma \tau}\right)^{t}\right)$ is selfadjoint on the one-particle space. It follows:

Lemma 5.3 The selfadjoint infinitesimal generators $M_{\sigma \tau}$ of the Lorentz transformations are the second quantization

$$
M_{\sigma \tau}=-i \int d \mu_{m}(p)\left(\left(\delta_{n n^{\prime}} p \wedge \partial_{p}+d(\omega)_{n n^{\prime}}^{t}\right)_{\sigma \tau} a_{n^{\prime}}^{*}(p)\right) a_{n}(p)
$$

of the operators $-i\left(\mathbb{I}_{d} p \wedge \partial_{p}+d(\omega)^{t}\right)_{\sigma \tau}$.

We now proceed as before with the momentum generators, inserting the partition of unity for the momenta via an $\vec{x}$-integration, and the partition of unity for the spin components via the sum eq. (5.8) over intertwiners. By partial integration in $p_{1}$, the operators $-i\left(\mathbb{I}_{d} p_{1} \wedge \partial_{p_{1}}+d(\omega)^{t}\right)$ acting on the creation operators $a^{*}\left(p_{1}\right)$ are shifted to the wave function $u\left(p_{1}\right) e^{i p_{1} x}$ where they act like

$$
-i\left(-p_{1} \wedge \partial_{p_{1}}+d(\omega)\right)\left(u\left(p_{1}\right) e^{i p_{1} x}\right)=\left(\left(i p_{1} \wedge \partial_{p_{1}}-i d(\omega)+x \wedge p_{1}\right) u\left(p_{1}\right)\right) e^{i p_{1} x} .
$$

The term $x \wedge p_{1}$ is treated exactly as before, and gives the expected contribution $\int d^{3} \vec{x}\left(x_{\sigma} \widetilde{T}_{0 \tau}-x_{\tau} \widetilde{T}_{0 \sigma}\right)$ to $M_{\sigma \tau}$. We are now going to compute the remaining term.

The infinitesimal version of the intertwining property eq. (5.7) is

$$
\left(p \wedge \partial_{p}\right) u(p)=(D(\Omega)+d(\omega)) u(p)
$$

therefore $i\left(p \wedge \partial_{p}-d(\omega)\right)_{\sigma \tau} u=i D\left(\Omega_{\sigma \tau}\right) u$ involves the infinitesimal Lorentz transformation $D\left(\Omega_{\sigma \tau}\right)$ of the intertwiner (which is the same as that of the field). Thus,

$$
M_{\sigma \tau}=\int d^{3} \vec{x}\left(x_{\sigma} \widetilde{T}_{0 \tau}-x_{\tau} \widetilde{T}_{0 \sigma}\right)+\Delta M_{\sigma \tau}
$$


where

$$
\Delta M_{\sigma \tau}=-\frac{1}{2} g^{M N} \int d^{3} \vec{x}:\left(D\left(\Omega_{\sigma \tau}\right) \phi\right)_{M} \stackrel{\leftrightarrow}{\partial_{0}} \phi_{N}:
$$

We now use Lemma B.1 in [16]: for a symmetric and conserved tensor $\Theta_{\rho \sigma}=\partial^{\mu}\left[X_{\rho \mu} \overleftrightarrow{\partial_{\sigma}}\right.$ $\left.Y+X_{\sigma \mu} \stackrel{\leftrightarrow}{\partial_{\rho}} Y\right]$, where $X_{\rho \mu}$ is anti-symmetric and both $X(x)$ and $Y(x)$ satisfy the KleinGordon equation, one has

$$
\int d^{3} \vec{x}\left(x_{\sigma} \Theta_{0 \tau}-x_{\tau} \Theta_{0 \sigma}\right)=2 \int d^{3} \vec{x} X_{\sigma \tau} \overleftrightarrow{\partial_{0}} Y
$$

Corollary 5.4 Under the assumption eq. (5.8), the total stress-energy tensor $\widetilde{T}_{\rho \sigma}+$ $\partial^{\mu} \Delta T_{\rho \sigma ; \mu}$ produces the correct generators of the Poincaré group eq. (5.1), where $\widetilde{T}_{\rho \sigma}$ is given by eq. (5.9) and

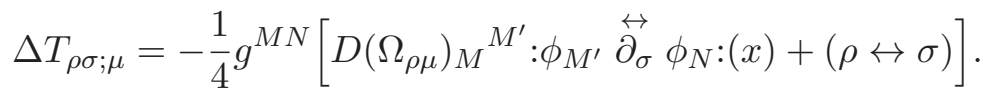

Notice that $\widetilde{T}_{\rho \sigma}$ and $\partial^{\mu} \Delta_{\rho \sigma ; \mu}$ are separately manifestly symmetric and conserved.

Example 5.5 For the massive Proca field of spin s, the standard intertwiner $u_{n}^{\mathrm{P}(s)}(p)=$ $\left(B_{p} E_{3}\right)^{\otimes s} T_{n}$ as in the proof of Proposition 3.4 fulfills eq. (5.8) with the metric $g=(-1)^{r} \eta^{\otimes r}$. The sign is due to the anti-isometric property of the embedding $E_{3}: \mathbb{R}^{3} \rightarrow \mathbb{R}^{4}$. The field $A^{\mathrm{P}(s)}$ transforms in the representation $D(\Lambda)=\Lambda^{\otimes s}$ of the Lorentz group. With $\left(\Omega_{\sigma \tau}\right)_{\mu}{ }^{\mu^{\prime}}=$ $\eta_{\tau \mu} \delta_{\sigma}^{\mu^{\prime}}-\eta_{\sigma \mu} \delta_{\tau}^{\mu^{\prime}}$, one gets

$$
\Delta M_{\sigma \tau}=-(-1)^{r} r \int d^{3} x: A_{\sigma \mu_{2} \ldots \mu_{s}}^{\mathrm{P}(s)} \stackrel{\leftrightarrow}{\partial_{0}} A_{\tau}^{\mathrm{P}(s) \mu_{2} \ldots \mu_{s}}:(x)
$$

and

$$
\Delta T_{\rho \sigma ; \mu}^{\mathrm{P}(s)}=-(-1)^{r} \frac{r}{2}\left[: A_{\rho \mu_{2} \ldots \mu_{s}}^{\mathrm{P}(s)} \stackrel{\leftrightarrow}{\partial_{\sigma}} A_{\mu}^{\mathrm{P}(s) \mu_{2} \ldots \mu_{s}}:(x)+(\rho \leftrightarrow \sigma)\right] .
$$

This gives the reduced stress-energy tensor eq. (5.2), found in [16] by a less systematic approach. Its main part $\widetilde{T}_{\rho \sigma}^{\mathrm{P}(s)}$ (without the derivative term) appeared already in Fierz' paper [8]. For the same Wigner representation $(m, s)$, none of the string-localized intertwiners $u^{(s, r)}$ fulfills eq. (5.8) separately. They must be combined, in a manner similar to the infinite-spin case below. In this case, Corollary 5.4 gives the regular stress-energy tensor eq. (5.3).

\subsection{Proof of Proposition 5.1}

In order to prove Proposition 5.1, we apply the prescription of the preceding subsection. By Corollary 5.4, we need to fulfill eq. (5.8) with intertwiners of string-localized fields. For the infinite-spin representations, the representation space $\mathcal{H}_{d}$ is $L^{2}\left(\kappa S^{1}\right)$ with $d \mu_{\kappa}(\vec{k})=\frac{d \varphi}{2 \pi}$, hence $\delta_{n_{1} n_{2}}$ is replaced by $\delta_{\kappa}\left(\vec{k}_{1}, \vec{k}_{2}\right)=2 \pi \cdot \delta_{2 \pi}\left(\varphi_{1}-\varphi_{2}\right)$. 
Lemma 5.6 The completeness relation eq. (5.8) is fulfilled by

$$
\sum_{r \geq 0}(-1)^{r} \eta^{\mu_{1} \nu_{1}} \ldots \eta^{\mu_{r} \nu_{r}} U_{\mu_{1} \ldots \mu_{r}}^{\kappa(r)}(e, p)\left(\vec{k}_{1}\right) \overline{U_{\nu_{1} \ldots \nu_{r}}^{\kappa(r)}(e, p)\left(\vec{k}_{2}\right)}=\delta_{\kappa}\left(\vec{k}_{1}, \vec{k}_{2}\right) .
$$

Proof. By Proposition 2.9(ii), the left-hand side equals $u^{\kappa(0)}(e, p)\left(\vec{k}_{1}\right) \overline{u^{\kappa(0)}(e, p)\left(\vec{k}_{2}\right)}$ times

$$
\left(\sum_{r \in \mathbb{Z}} e^{-i r\left(\varphi_{1}-\varphi_{2}\right)}\right)=2 \pi \delta_{2 \pi}\left(\varphi_{1}-\varphi_{2}\right)=\delta_{\kappa}\left(\vec{k}_{1}, \vec{k}_{2}\right)
$$

and at $\vec{k}_{1}=\vec{k}_{2}$ the factor is $=1$ because $u^{\kappa(0)}(e, p)(\vec{k})$ is a complex phase.

Remark 5.7 One sees why it is crucial that the two strings in eq. (5.12) are equal: otherwise the phase factors $u^{\kappa(0)} \overline{u^{\kappa(0)}}$ would fail to cancel. It is also essential that $u^{\kappa(0)}$ is a function (Proposition 2.1), since otherwise the product of distributions involving $\frac{e}{(e p)_{+}}$and $\frac{e}{\frac{e}{(e p)_{+}}}=\frac{e}{(e p)_{-}}$were ill-defined. Because the factors $u^{\kappa(0)}$ only appear through the inductive limit appendix A, cf. Remark 4.5, they are absent in the case of finite spin, and the analogous requirement of coinciding strings may be dropped, as in eq. (5.3).

We can thus apply Corollary 5.4 mutatis mutandis. Apart from the specific partition of unity eq. (5.12), the only change is the dependence of the intertwiners on $e$, which are also transformed along with the Lorentz tensors by $D(\Lambda)$, specifying eq. (5.7) as

$$
U^{\kappa(r)}(e, \Lambda p)=\left(\Lambda^{\otimes r} \otimes d_{\kappa}\left(W_{\Lambda, p}\right)\right) U^{\kappa(r)}\left(\Lambda^{-1} e, p\right) \quad\left(\Lambda \in \mathrm{SO}(1,3)_{+}^{\uparrow}\right) .
$$

Therefore, $D\left(\Omega_{\sigma \tau}\right)$ contains, besides the infinitesimal Lorentz matrices, the additional term $-\left(e \wedge \partial_{e}\right)_{\sigma \tau}$, and $\Delta T$ is a sum of two terms:

$$
\Delta T_{\rho \sigma ; \mu}^{\kappa}(e, x)=\Delta_{1} T_{\rho \sigma ; \mu}^{\kappa}(e, x)+\Delta_{2} T_{\rho \sigma ; \mu}^{\kappa}(e, x)
$$

where

$$
\Delta_{1} T_{\rho \sigma ; \mu}^{\kappa}(e, x)=-\sum_{r \geq 0}(-1)^{r} \frac{r}{2}\left[: \Phi_{\rho \mu_{2} \ldots \mu_{r}}^{\kappa(r)}(e) \overleftrightarrow{\partial_{\sigma}} \Phi_{\mu}^{\kappa(r) \mu_{2} \ldots \mu_{r}}(e):(x)+(\rho \leftrightarrow \sigma)\right]
$$

similar as in Example 5.5, and

$$
\Delta_{2} T_{\rho \sigma ; \mu}^{\kappa}(e, x)=-\sum_{r \geq 0} \frac{(-1)^{r}}{8}\left[: \Phi_{\mu_{1} \ldots \mu_{r}}^{\kappa(r)}(e)\left(e \wedge \stackrel{\leftrightarrow}{\partial_{e}}\right)_{\rho \mu} \stackrel{\leftrightarrow}{\partial_{\sigma}} \Phi^{\kappa(r) \mu_{1} \ldots \mu_{r}}(e):(x)+(\rho \leftrightarrow \sigma)\right]
$$

With the computation of $\widetilde{T}$, coinciding with the expression displayed in eq. (5.5), and the specification of $\Delta T=\Delta_{1} T+\Delta_{2} T$, the proof of Proposition 5.1 is complete.

Remark 5.8 Derivatives w.r.t. e seem to mix $\Phi^{\kappa(r)}$ with $\Phi^{\kappa(r \pm 1)}$ by eq. (2.36), but because eq. (2.36) involves a symmetrization, we cannot simply write $\left(e \wedge \partial_{e}\right) \Phi^{\kappa(r)}$ as a combination of $\Phi^{\kappa(r \pm 1)}$. Recall, however, that the fields $\Phi^{\kappa(r)}(e)$ are simultaneously defined for all $e$ on the same Hilbert space, and the derivative w.r.t. e does not change the localization. Therefore, each $T^{\kappa(r)}$ in eq. (5.5) is well-defined. 
The potential problems due to the infinite summation over $r$ in eq. (5.5) will be discussed in the next section.

The same general strategy outlined in section 5.1 applies to conserved currents of complex fields. In the case of infinite spin, the partition of unity eq. (5.12) inserted into the charge operator

$$
Q=\int d \mu_{0}(p) d \mu_{\kappa}(\vec{k})\left(a^{*}(p, \vec{k}) a(p, \vec{k})-b^{*}(p, \vec{k}) b(p, \vec{k})\right)
$$

gives rise to the current

$$
J_{\rho}^{\kappa}(e, x)=i \sum_{r \geq 0}(-1)^{r}: \Phi_{\mu_{1} \ldots \mu_{r}}^{\kappa(r) *}(e) \stackrel{\leftrightarrow}{\partial_{\rho}} \Phi^{\kappa(r) \mu_{1} \ldots \mu_{r}}(e):(x)
$$

Remark 5.9 Infinite-spin fields admit no subalgebra of compactly localized observables ("field strengths" or currents) whose charged sectors they would generate from the vacuum [12, 13]. Therefore, "neutral" operators like the current densities or stress-energy tensor cannot be point-localized as in the massive case; the localization on a pair of opposite strings seems to be the best that is possible.

\subsection{Properties of the infinite-spin stress-energy tensor}

We present here some qualitative material that helps to assess the mathematical nature of fields like the infinite-spin stress-energy tensor eq. (5.5). The rigorous analytical treatment is beyond the scope of this article.

\subsubsection{Matrix elements}

For a generic one-particle state $\Psi=\int d \mu_{0}(p) d \mu_{\kappa}(\vec{k}) \psi(p, \vec{k}) a^{*}(p, \vec{k}) \Omega$, we compute matrix elements

$$
\left(\Psi, \Phi^{\kappa(r)}(e, x) \Omega\right)=\int d \mu_{0}(p) e^{i p x}\left(\psi(p), U^{\kappa(r)}(e, p)\right)_{\kappa}
$$

where $(\cdot, \cdot)_{\kappa}$ is the scalar product of $L^{2}\left(\kappa S^{1}\right)$. Let $R_{e, p}\left(\begin{array}{c}\cos \alpha_{e, p} \\ \sin \alpha_{e, p}\end{array}\right)$ parametrize the 1-2-part of $B_{p}^{-1} q_{e}(p)$, so that $u^{\kappa(0)}(e, p)(\vec{k})=e^{-i\left(q_{e} E_{p}(\vec{k})\right)}=e^{i \kappa R_{e, p} \cos \left(\varphi-\alpha_{e, p}\right)}$.

For $\psi^{n}(p, \vec{k})=\psi(p) e^{i n \varphi}$, the $\vec{k}$-integration produces Bessel functions eq. (3.9). Thus, with $\mathcal{E}_{ \pm}(e, p)=J_{e}(p) E_{p} \varepsilon_{ \pm}$as in Proposition 2.9

$$
\left(\psi^{n}(p), U^{\kappa(r)}(e, p)\right)_{\kappa}=(-1)^{r} \overline{\psi(p)} \omega^{\kappa}(e, p) \sum_{ \pm} J_{n \pm r}\left(\kappa R_{e, p}\right) e^{-i(n \pm r)\left(\alpha_{e, p}-\frac{\pi}{2}\right)} \cdot \mathcal{E}_{ \pm}(e, p)^{\otimes r}
$$

For $r=0$, the sum of two terms is replaced by $J_{n}\left(\kappa R_{e, p}\right) e^{-i n\left(\alpha_{e, p}-\frac{\pi}{2}\right)}$.

This formula is not particularly useful, but it shows that there is no correlation between $r$ and $n$ (cf. Remark 2.10), and that infinite sums over $r$, as in the stress-energy tensor or the current, are potentially dangerous, as already pointed out in Remark 5.2. 
Let us exemplarily investigate this issue in various situations: matrix elements, twopoint function, and commutators of the stress-energy tensor or the current. In order to simplify the presentation, we consider the scalar Wick square

$$
W^{\kappa}(e, x)=\sum_{r=0}^{\infty}(-1)^{r}: \Phi^{\kappa(r) \mu_{1} \ldots \mu_{r}}(e) \Phi_{\mu_{1} \ldots \mu_{r}}^{\kappa(r)}(e):(x),
$$

in which the characteristic features of the infinite sum can be seen as well. For the actual stress-energy tensor, one basically has to insert polynomial factors of $p$ corresponding to the derivatives $\overleftrightarrow{\partial_{\rho}} \stackrel{\leftrightarrow}{\partial_{\sigma}}$, and add a similar contribution from $\Delta T$.

We compute matrix elements of the Wick square, for simplicity in one-particle states $\Psi_{i}$ with wave functions $\psi_{i}^{0}(p)$ (i.e., $\left.n=0\right)$ :

$$
\begin{aligned}
\left(\Psi_{1}^{0}, W^{\kappa}(e, x) \Psi_{2}^{0}\right)= & 2 \int d \mu_{0}\left(p_{1}\right) d \mu_{0}\left(p_{2}\right) e^{i\left(p_{1}-p_{2}\right) x} \overline{\psi_{1}\left(p_{1}\right)} \psi_{2}\left(p_{2}\right) \cdot \omega^{\kappa}\left(e, p_{1}\right) \overline{\omega^{\kappa}\left(e, p_{2}\right)} \\
& \cdot \sum_{\nu \in \mathbb{Z}} e^{-i \nu\left(\alpha_{e, p_{1}}-\alpha_{e, p_{2}}\right)} J_{\nu}\left(\kappa R_{e, p_{1}}\right) J_{\nu}\left(\kappa R_{e, p_{2}}\right) .
\end{aligned}
$$

Similar expressions with $J_{\nu+n_{1}} J_{\nu+n_{2}}$ hold for matrix elements in states with $n_{i} \neq 0$, or for matrix elements between the vacuum and two-particle states.

The point is that the sum over $r \in \mathbb{Z}$ is absolutely convergent thanks to the CauchySchwartz inequality applied to the square-summability of the Bessel functions:

$$
\sum_{\nu \in \mathbb{Z}} J_{\nu}(x)^{2}=1
$$

Together with Wick's theorem for matrix elements between multi-particle states, this observation supports our

Conjecture 5.10 The Wick square eq. (5.15) and likewise the stress-energy tensor eq. (5.5) and the current eq. (5.14) have finite matrix elements in states of finite particle number and finite energy. Because such states are dense in the Fock space, these fields exist as quadratic forms with a dense domain.

\subsubsection{Two-point functions and vacuum fluctuations}

The two-point function of $W^{(\kappa)}$ is a double sum over $r$ and $r^{\prime}$ of the fully contracted squares of two-point functions $\left(\Omega, \Phi_{\underline{\mu}}^{\kappa(r)}(x) \Phi_{\underline{\nu}}^{\kappa\left(r^{\prime}\right)}(y) \Omega\right)$ given in Proposition 3.2.

If $R_{e, e^{\prime}, p}\left(\begin{array}{c}\cos \alpha_{e, e^{\prime}, p} \\ \sin \alpha_{e, e^{\prime}, p}\end{array}\right)$ parametrizes the 1-2-part of $B_{p}^{-1}\left(q_{e}(p)-q_{e^{\prime}}(p)\right)$, then

$$
\begin{gathered}
\left(\Omega, W^{\kappa}(e, x) W^{\kappa}\left(e^{\prime}, x^{\prime}\right) \Omega\right)=2 \int d \mu_{0}\left(p_{1}\right) d \mu_{0}\left(p_{2}\right) e^{-i\left(p_{1}+p_{2}\right)\left(x-x^{\prime}\right)} \prod_{i=1,2} \overline{\omega^{\kappa}\left(e, p_{i}\right)} \omega^{\kappa}\left(e^{\prime}, p_{i}\right) . \\
\cdot \sum_{\nu, \nu^{\prime} \in \mathbb{Z}}(-1)^{\nu-\nu^{\prime}} e^{i\left(\nu+\nu^{\prime}\right)\left(\alpha_{e, e^{\prime}, p_{1}}-\alpha_{e, e^{\prime}, p_{2}}\right)} J_{\nu+\nu^{\prime}}\left(\kappa R_{e, e^{\prime}, p_{1}}\right) J_{\nu+\nu^{\prime}}\left(\kappa R_{e, e^{\prime}, p_{2}}\right) .
\end{gathered}
$$

The problem is that the double sum may not exist, because the square-summability and Cauchy-Schwartz argument (as for the matrix elements) does not apply: the convolution product of square-summable sequences need not be square-summable.

Of course, smearing with test functions does not help. This supports our 
Conjecture 5.11 The two-point functions of $W^{\kappa}, T_{\rho \sigma}^{\kappa}$, and $J_{\rho}^{\kappa}$ do not exist.

Mathematically, this means that the stress-energy tensor does not exist as an operatorvalued distribution with a stable domain containing the vacuum vector, as required by the Wightman axioms. In physical terms, the divergence of the two-point function signals infinitely strong vacuum fluctuations. Stress-energy tensors that exist as quadratic forms (Conjecture 5.10), but not as Wightman fields (Conjecture 5.11), occur also for generalized free fields [6]. Here, the vacuum fluctations are also divergent, but not because of the infinitely degenerate spin component, but because a continuous mass distribution cannot be "square-summable".

\subsubsection{Commutators}

We have seen that the decisive difference between the "good" behaviour of matrix elements and the "bad" behaviour of two-point functions is due to the summation structure. Let us therefore study the commutator of the Wick square with a field just under this aspect.

The summation structure of the commutator is the same as that of a matrix element $\left(\Psi, W^{\kappa} \Phi^{\kappa(r)} \Omega\right)$ with a one-particle state. Choose for simplicity $r=0$, and $\Psi^{n}$ of helicity $n$ as in section 5.3.1. Then, one computes

$$
\begin{aligned}
& \left(\Psi^{n}, W^{\kappa}(e, x) \Phi^{\kappa(0)}\left(e^{\prime}, x^{\prime}\right) \Omega\right)= \\
& =2 \int d \mu_{0}\left(p_{1}\right) d \mu_{0}\left(p_{2}\right) e^{i p_{1} x} e^{-i p_{2}\left(x-x^{\prime}\right)} \cdot \overline{\psi^{n}\left(p_{1}\right)} \cdot \omega^{\kappa}\left(e, p_{1}\right) \overline{\omega^{\kappa}\left(e, p_{2}\right)} \omega^{\kappa}\left(e^{\prime}, p_{2}\right) . \\
& \quad \cdot \sum_{\nu \in \mathbb{Z}} i^{n} e^{-i(n+\nu) \alpha_{e, p_{1}}} e^{i \nu \alpha_{e e^{\prime}, p_{2}} J_{\nu+n}\left(\kappa R_{e, p_{1}}\right) J_{\nu}\left(\kappa R_{e, e^{\prime}, p_{2}}\right) .}
\end{aligned}
$$

This sum is absolutely convergent, as for the matrix elements above. The same expression with a different $i \varepsilon$ prescription (hidden in the argument $R_{e, e^{\prime}, p_{2}}$ of the Bessel function) holds for the matrix element $\left(\Psi, \Phi^{\kappa(r)} W^{\kappa} \Omega\right)$, and hence the sum also converges for the commutator. This sketch of an argument supports our

Conjecture 5.12 The commutators of $W^{\kappa}, T_{\rho \sigma}^{\kappa}$, and $J_{\rho}^{\kappa}$ with the linear fields $\Phi_{\mu_{1} \ldots \mu_{r}}^{\kappa(r)}$ exist and can be defined as derivations on the algebra generated by smeared fields.

In view of Conjecture 5.11, this property would rescue the stress-energy tensor as a "good" physical quantity. Namely, the prime role of the stress-energy tensor in quantum field theory is to generate infinitesimal Poincaré transformations via commutators. Of course, other technical issues remain concerning the convergence of the commutator with a smeared stress-energy tensor when the smearing functions becomes constant in space and sharp in time.

More interestingly, Conjecture 5.12 could also secure the existence of the perturbative expansion of a coupling of infinite-spin matter to linearized gravity via its stress-energy tensor, because this expansion is a series in retarded commutators.

More detailed investigations of these issues are beyond the scope of this paper. 


\subsubsection{Thermal states: equation of state and equipartition}

Further interesting quantities to study are the energy density and the pressure in thermal equilibrium.

The computation of thermal expectation values of quadratic fields :XY:(x) is most easily done by first considering $\omega_{\beta}\left(X(x) Y\left(x^{\prime}\right)\right)$ at $x \neq x^{\prime}$ and using the KMS condition in momentum space (e.g., [19], eq. (16)). It determines the thermal two-point kernel on the negative mass shell by "detailed balance":

$$
M_{m, \beta}^{X, Y}(-p)=e^{-\beta p^{0}} \cdot M_{m, \beta}^{Y, X}(p) .
$$

Then one exploits the fact that the commutator is the same in the vacuum and in the thermal state, hence $M_{m, \mathrm{vac}}^{X, Y}(p)=M_{m, \beta}^{X, Y}(p)-M_{m, \beta}^{Y, X}(-p)$. This implies

$$
M_{m, \beta}^{X, Y}(p)=\frac{1}{1-e^{-\beta p^{0}}} \cdot M_{m, \mathrm{vac}}^{X, Y}(p)
$$

on the positive mass shell. Subtracting the vacuum expectation value, one gets

$$
\omega_{\beta}\left(: X(x) Y\left(x^{\prime}\right):\right)=\int d \mu_{m}(p) \frac{1}{e^{\beta p^{0}}-1}\left[M_{m, \mathrm{vac}}^{X, Y}(p) e^{-i p(x-y)}+M_{m, \mathrm{vac}}^{Y, X}(p) e^{i p(x-y)}\right] .
$$

Here, one can put $x=x^{\prime}$, and thus obtains the thermal expectation value of :XY:(x) from the vacuum two-point kernels.

This very efficient method reduces the computations of thermal expectation values to the inspection of the vacuum kernels, without any computation of partition functions in finite volume. It immediately gives the thermal energy density $\varepsilon=\omega_{\beta}\left(T_{00}^{\mathrm{red}(s)}\right)$ and the pressure $p=\omega_{\beta}\left(T_{i i}^{\mathrm{red}(s)}\right)$ of massive matter of finite spin

$$
\varepsilon=\frac{(2 s+1)}{2 \pi^{2} \beta^{4}} \cdot I_{+}(\beta m), \quad 3 p=\frac{(2 s+1)}{2 \pi^{2} \beta^{4}} \cdot I_{-}(\beta m),
$$

where $I_{ \pm}(x)=\int_{0}^{\infty} \frac{u^{3} d u}{e^{\sqrt{u^{2}+x^{2}}}-1}\left(\frac{\sqrt{u^{2}+x^{2}}}{u}\right)^{ \pm 1}$. The manifest factor $2 s+1$ reflects the law of equipartition. The result is independent of the choice of the stress-energy tensor, because KMS states are translation invariant, hence the derivative terms by which various stressenergy tensors differ, do not contribute. Interestingly, the individual contributions from $t^{(s, r)}$ in eq. (5.3) depend on $e_{1}$ and $e_{2}$, while only their sum is independent of the strings. E.g., for $s=1$, the contributions are $1-m^{2}\left(q_{e_{1}} q_{e_{2}}\right)$ from $r=0$ and $2+m^{2}\left(q_{e_{1}} q_{e_{2}}\right)$ from $r=1$. In the Pauli-Lubanski limit, each contribution from $t^{(s, r)}$ converges to zero (because of the factor $F_{s}(1)$ in eq. (4.3)), but their sum diverges as $2 s+1$ (because of eq. (5.17)). The total energy density per degree of freedom and the pressure per degree of freedom remain finite, and obey the usual massless equation of state.

The string-localized stress-energy tensor $T^{(s) m=0}$ of massless fields of finite helicity $|h|>0$ gives the factor 2 , as expected. At $m=0$, the finite values $I_{+}(0)=I_{-}(0)=$ $\frac{\pi^{4}}{15}$ reproduce the Stefan-Boltzmann law and the massless equation of state $p(\varepsilon)=\frac{1}{3} \varepsilon$. (Interestingly, while the trace of the reduced stress-energy tensor is non-zero and not even defined at $m=0$, its thermal expectation value vanishes in the limit $m \rightarrow 0$ ). 
For the infinite-spin stress-energy tensor, the contribution from each $T^{\kappa(r)}$ is 2 (resp. 1 for $r=0$ ). Thus, the sum over $r$ diverges as $2 r+1$, confirming the heuristic expectation. Wigner argued in [33] that this need not imply that infinite-spin matter must be unphysical, because it might never reach thermal equilibrium. Of course, this question cannot be physically addressed without a dynamical model for the coupling to ordinary matter. E.g., Schroer [24] argues that infinite-spin matter cannot couple to ordinary matter because there is no interaction Lagrangean that yields a string-independent action, as is needed to preserve causality in the quantum perturbation theory [16]. Thus infinite-spin matter is "inert", and has no mechanism to approach thermal equilibrium at all.

\section{Conclusion}

We have "liberated quantum field theory from its classical crutches" (in the words of P. Jordan) by finding a construction scheme for covariant quantum stress-energy tensors that does not refer to a classical action. The method is applicable to arbitrary (in this paper: integer or infinite) spin. Auxiliary fields implementing higher-spin constraints, negative probability states, and compensating ghosts never appear.

Instead, the prescription is based on Wigner's unitary representation theory of the Poincaré group and Weinberg's construction of covariant quantum fields with the help of intertwiners whose analytic properties entail the localization properties of the fields.

The achieved stress-energy tensors are not unique, depending on a choice of intertwiners fulfilling the localizing completeness relation eq. (5.8). However, their densities all differ by "irrelevant derivatives" in the sense that they all produce the same Poincaré generators when integrated over space at a fixed time. Even for low spin, our "reduced" stress-energy tensors (Example 5.5) differ from the canonical or Hilbert stress-energy tensors by irrelevant derivative terms.

We applied this method in the case of the infinite-spin representations, where the best possible localization is on strings of the form $S_{e}(x)=x+\mathbb{R}_{+} \cdot e$. In this case, the completeness relation eq. (5.8) requires an infinite direct sum of representations of the Lorentz group, which causes the stress-energy tensor to be an infinite sum of quadratic expressions in the corresponding string-localized fields. We sketched in section 5.3 the ensuing analytical implications (problems of convergence) with indications for "one bad and two good" features.

The good features are that matrix elements and commutators of the stress-energy tensor are well-behaved, while its correlations functions suffer from infinite vacuum fluctuations (the price of infinite spin).

The involved string-localized fields are defined on the Fock space over Wigner's infinitespin representation. We constructed these fields as Pauli-Lubanski limits of tensor fields of increasing spin and decreasing mass with fixed Pauli-Lubanski parameter $\kappa^{2}=m^{2} s(s+1)$. Although it is not needed for the determination of the stress-energy tensor, this approximation is of some interest of its own. E.g., it exhibits how the dynamical coupling between escort fields $A^{(s, r)}$ of different $r$, that goes to zero with the mass at fixed $s$, remains stable (proportional to $\kappa$ ) when the spin increases. This may also play a role in higher spin theories. 


\section{Acknowledgments}

I am grateful for invitations to the Universidade de Juiz de Fora, where this project has started, and to the University of York, where parts of it have been done. I thank Bert Schroer and Jens Mund for helpful discussions and Jakob Yngvason for his encouraging interest. This work had been impossible without their groundbreaking work on the infinitespin representations. I thank the referee for pointing out ref. [14].

\section{A Pauli-Lubanski limit of Wigner representations}

We give a non-techical presentation of the Pauli-Lubanski limit of the (one-particle) Wigner representations. For a more rigorous treatment, see [14].

The standard reference vector of the massive Wigner representation $(m, s)$ is $p_{m}=$ $(m, 0,0,0)$. Its stabilizer group is $\operatorname{Stab}\left(p_{m}\right)=\mathrm{SO}(3) \subset \mathrm{SO}(1,3)$. We denote its generators $L_{i}$ as usual, and $K_{i}$ the generators of the boosts. The reference vector for a massless Wigner representation is $p_{0}=(1,0,0,1)^{t}$. We approximate it by massive vectors $p_{\tau}=$ $m(\cosh \tau, 0,0, \sinh \tau)^{t}$ with $e^{\tau}=\frac{2}{m}$ in the limit $m \rightarrow 0$. Let $B^{\tau}$ be the Lorentz 3-boost such that $B^{\tau} p_{m}=p_{\tau}$. The stabilizer group of $p_{\tau}$ is $\operatorname{Stab}\left(p_{\tau}\right)=B^{\tau} \operatorname{SO}(3) B^{\tau-1}$.

For the generators of $\operatorname{Stab}\left(p_{\tau}\right)$ one computes $L_{3}^{\tau}:=B^{\tau} L_{3} B^{\tau-1}=L_{3}$ and $L_{i}^{\tau}:=$ $B^{\tau} L_{i} B^{\tau-1}=\cosh \tau L_{i}+\sinh \tau K_{i}$ for $i=1,2$. Thus, in $\operatorname{so}(1,3)$

$$
\lim _{\tau \rightarrow \infty} 2 e^{-\tau} L_{i}^{\tau}=Q_{i}
$$

are the "translation" generators of $\operatorname{Stab}\left(p_{0}\right)=\mathrm{E}(2)$, while $L_{3}$ is the generator of the rotations in $\mathrm{E}(2)$.

Now, we choose the unitary representation of $\mathrm{SO}(3)$ with standard orthonormal basis $|s, n\rangle \in \mathcal{H}_{s}$ for each spin, for which $L_{3}|s, n\rangle=n \cdot|s, n\rangle$ and $L_{ \pm}|s, n\rangle=\sqrt{s(s+1)-n(n \pm 1)}$. $|s, n \pm 1\rangle$. For increasing $s$, we isometrically embed $\mathcal{H}_{s} \rightarrow \mathcal{H}_{s+1}$ by $|s, n\rangle \mapsto|s+1, n\rangle$. The Pauli-Lubanski limit is the inductive limit of the representations of the generators $2 e^{-\tau} L_{i}^{\tau}$ and $L_{3}^{\tau}$ in this sequence of representations as $s \rightarrow \infty$, while $m^{2} s(s+1)=\kappa^{2}$ is constant. Thus, $2 e^{-\tau}=m=\kappa(s(s+1))^{-\frac{1}{2}}$, hence

$$
2 e^{-\tau} L_{ \pm}^{\tau}|s, n\rangle=\kappa\left(1-\frac{n(n \pm 1)}{s(s+1)}\right)^{\frac{1}{2}} \cdot|s, n \pm 1\rangle, \quad L_{3}^{\tau}|s, n\rangle=n \cdot|s, n\rangle .
$$

In the inductive limit, this becomes the representation $D_{\kappa}$ of $\mathrm{E}(2)$ :

$$
Q_{ \pm}|n\rangle=\kappa \cdot|n \pm 1\rangle, \quad L_{3}|n\rangle=n \cdot|n\rangle .
$$

(Representing $|n\rangle \in \mathcal{H}_{\kappa}=L^{2}\left(\kappa S^{1}\right)$ by the wave function $\psi_{n}(\varphi)=e^{i n \varphi}, Q_{ \pm}$act by multiplication with $\kappa e^{ \pm i \varphi}$, hence $\vec{Q}$ act by multiplication with $\vec{k}=\kappa(\cos \varphi, \sin \varphi)$.)

Once the representation $d$ of the respective stabilizer group is specified, the corresponding induced Wigner representation of the Poincaré group is defined on $L^{2}\left(H_{m}, \mathcal{H}_{d}\right)$. The translations act on wavefunctions $\psi(p)$ with values in $\mathcal{H}_{d}$ by multiplication with $e^{i p x}$, and the Lorentz transformations act by

$$
(U(\Lambda) \psi)(p)=d\left(W_{\Lambda, \Lambda^{-1} p}\right) \psi\left(\Lambda^{-1} p\right),
$$


where $W_{\Lambda, p}=B_{\Lambda p}^{-1} \Lambda B_{p}$ is the Wigner "rotation" in the stabilizer group of the respective reference vector $p_{0}$. It depends on the choice of the standard "boosts" $B_{p}$ that take $p_{0}$ to $p$, but the dependence is a unitary equivalence of $U$. This unitary equivalence acts on $L^{2}\left(H_{m}, \mathcal{H}_{d}\right)$ as a multiplication with a function $H_{m} \rightarrow U(\mathcal{H})$, and is of course irrelevant for abstract properties.

The inductive limit of representations of the stabilizer groups, outlined before, naturally extends to the induced representations of the Poincaré group.

Open Access. This article is distributed under the terms of the Creative Commons Attribution License (CC-BY 4.0), which permits any use, distribution and reproduction in any medium, provided the original author(s) and source are credited.

\section{References}

[1] X. Bekaert and J. Mourad, The Continuous spin limit of higher spin field equations, JHEP 01 (2006) 115 [hep-th/0509092] [INSPIRE].

[2] X. Bekaert, J. Mourad and M. Najafizadeh, Continuous-spin field propagator and interaction with matter, arXiv:1710.05788 [INSPIRE].

[3] X. Bekaert and E.D. Skvortsov, Elementary particles with continuous spin, Int. J. Mod. Phys. A 32 (2017) 1730019 [arXiv:1708.01030] [INSPIRE].

[4] R. Brunetti, D. Guido and R. Longo, Modular localization and Wigner particles, Rev. Math. Phys. 14 (2002) 759 [math-ph/0203021] [INSPIRE].

[5] D. Buchholz and K. Fredenhagen, Locality and the Structure of Particle States, Commun. Math. Phys. 84 (1982) 1 [INSPIRE].

[6] M. Dütsch and K.-H. Rehren, Generalized free fields and the AdS-CFT correspondence, Ann. Inst. H. Poincare 4 (2003) 613 [math-ph/0209035].

[7] A. Erdélyi, Higher Transcendental Functions. Vol. I (Bateman Manuscript Project), McGraw-Hill, New York U.S.A. (1953).

[8] M. Fierz, Über die relativistische Theorie kräftefreier Teilchen mit beliebigem Spin, Helv. Phys. Acta 12 (1939) 3 [INSPIRE].

[9] J. Fröhlich, G. Morchio and F. Strocchi, Infrared problem and spontaneous breaking of the Lorentz group in QED, Phys. Lett. 89B (1979) 61 [INSPIRE].

[10] R. Gonzo, The infinite-spin representations of the Poincaré group, MSc Thesis, University of Padova, Padova Italy (2017).

[11] G. Grensing, Symmetric and Traceless Tensors on Minkowski Space, Rept. Math. Phys. 14 (1978) 19 [INSPIRE].

[12] C. Köhler, On the localization properties of quantum fields with zero mass and infinite spin, Ph.D. Thesis, University of Vienna, Vienna Austria (2015).

[13] R. Longo, V. Morinelli and K.-H. Rehren, Where Infinite Spin Particles Are Localizable, Commun. Math. Phys. 345 (2016) 587 [arXiv:1505.01759] [INSPIRE].

[14] A. McKerrell, Canonical representations for massless particles and zero-mass limits of the helicity representation, Proc. Roy. Soc. A 285 (1965) 287. 
[15] J. Mund, String-localized vector bosons without ghosts and indefinite metric: the example of massive $Q E D$, work in progress.

[16] J. Mund, K.-H. Rehren and B. Schroer, Helicity decoupling in the massless limit of massive tensor fields, Nucl. Phys. B 924 (2017) 699 [arXiv:1703.04407] [INSPIRE].

[17] J. Mund, K.-H. Rehren and B. Schroer, Relations between positivity, localization and degrees of freedom: The Weinberg-Witten theorem and the van Dam-Veltman-Zakharov discontinuity, Phys. Lett. B 773 (2017) 625 [arXiv: 1703.04408] [INSPIRE].

[18] J. Mund, B. Schroer and J. Yngvason, String-localized quantum fields and modular localization, Commun. Math. Phys. 268 (2006) 621 [math-ph/0511042] [INSPIRE].

[19] J. Mund, B. Schroer and J. Yngvason, String localized quantum fields from Wigner representations, Phys. Lett. B 596 (2004) 156 [math-ph/0402043] [INSPIRE].

[20] M. Plaschke and J. Yngvason, Massless, String Localized Quantum Fields for Any Helicity, J. Math. Phys. 53 (2012) 042301 [arXiv:1111.5164] [INSPIRE].

[21] V.O. Rivelles, Remarks on a Gauge Theory for Continuous Spin Particles, Eur. Phys. J. C 77 (2017) 433 [arXiv: 1607.01316] [INSPIRE].

[22] B. Schroer, A Hilbert space setting for interacting higher spin fields and the Higgs issue, Found. Phys. 45 (2015) 219 [arXiv:1407.0360] [INSPIRE].

[23] B. Schroer, Beyond gauge theory: positivity and causal localization in the presence of vector mesons, Eur. Phys. J. C 76 (2016) 378 [arXiv:1601.04528] [INSPIRE].

[24] B. Schroer, Wigner's infinite spin representations and inert matter, Eur. Phys. J. C 77 (2017) 362 [arXiv: 1601.02477] [InSPIRE].

[25] P. Schuster and N. Toro, On the Theory of Continuous-Spin Particles: Wavefunctions and Soft-Factor Scattering Amplitudes, JHEP 09 (2013) 104 [arXiv:1302.1198] [INSPIRE].

[26] P. Schuster and N. Toro, On the Theory of Continuous-Spin Particles: Helicity Correspondence in Radiation and Forces, JHEP 09 (2013) 105 [arXiv:1302.1577] [INSPIRE].

[27] P. Schuster and N. Toro, A Gauge Field Theory of Continuous-Spin Particles, JHEP 10 (2013) 061 [arXiv:1302.3225] [INSPIRE].

[28] S. Weinberg, The Quantum Theory of Fields, Vol. I, Cambridge University Press, Cambridge U.K. (1995).

[29] S. Weinberg, Feynman Rules for Any Spin, Phys. Rev. B 133 (1964) 1318.

[30] S. Weinberg, Feynman Rules for Any Spin. II. Massless Particles, Phys. Rev. B 134 (1964) 882.

[31] S. Weinberg and E. Witten, Limits on massless particles, Phys. Lett. B 96 (1980) 59 [INSPIRE].

[32] E.P. Wigner, On Unitary Representations of the Inhomogeneous Lorentz Group, Annals Math. 40 (1939) 149 [INSPIRE].

[33] E.P. Wigner, Relativistische Wellengleichungen, Z. Phys. 124 (1948) 665.

[34] J. Yngvason, Zero-mass infinite spin representations of the Poincaré group and quantum field theory, Commun. Math. Phys. 18 (1970) 195 [INSPIRE]. 